\title{
The science case for an orbital mission to Uranus: Exploring the origins and evolution of ice giant planets
}

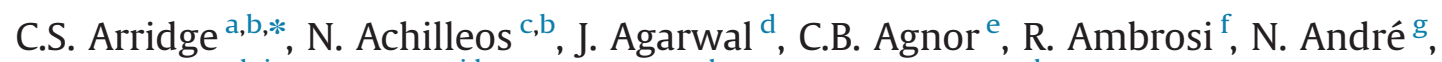
S.V. Badman ${ }^{\text {h,i }}$, K. Baines ${ }^{\mathrm{j}, \mathrm{k}}$, D. Banfield ${ }^{1}$, M. Barthélémy ${ }^{\mathrm{m}, \mathrm{br}}$, M.M. Bisi ${ }^{\mathrm{n}}$, J. Blum ${ }^{ }$, T. Bocanegra-Bahamon ${ }^{\mathrm{P}}$, B. Bonfond ${ }^{\mathrm{q}}, \mathrm{C}$. Bracken ${ }^{\mathrm{r}}, \mathrm{P}$. Brandt ${ }^{\mathrm{s}}, \mathrm{C}$. Briand ${ }^{\mathrm{t}}, \mathrm{C}$. Briois ${ }^{\mathrm{u}}$, S. Brooks ${ }^{j}$, J. Castillo-Rogez ${ }^{\mathrm{j}}$, T. Cavalié ${ }^{\mathrm{v}}$, B. Christophe ${ }^{\mathrm{w}}$, A.J. Coates ${ }^{\mathrm{a}, \mathrm{b}}$, G. Collinson ${ }^{\mathrm{x}}$, J.F. Cooper ${ }^{\mathrm{x}}$, M. Costa-Sitja ${ }^{\mathrm{y}}$, R. Courtin ${ }^{\mathrm{t}}$, I.A. Daglis ${ }^{\mathrm{z}}$, I. de Pater $^{\mathrm{aa}}$, M. Desai $^{\mathrm{ab}}$, D. Dirkx $^{\mathrm{p}}$, M.K. Dougherty ${ }^{\mathrm{ac}}$, R.W. Ebert ${ }^{\mathrm{ab}}$, G. Filacchione ${ }^{\mathrm{ad}}$, L.N. Fletcher ${ }^{\mathrm{ae}}$, J. Fortney ${ }^{\text {af }}$, I. Gerth ${ }^{\mathrm{p}}$, D. Grassi ${ }^{\text {ad }}$, D. Grodent ${ }^{\text {q }}$, E. Grün ${ }^{\text {ag,ah }}$, J. Gustin ${ }^{\mathrm{q}}$, M. Hedman ${ }^{\text {ai }}$, R. Helled ${ }^{\mathrm{aj}}$, P. Henri ${ }^{\mathrm{u}}$, S. Hess ${ }^{\text {ak }}$, J.K. Hillier ${ }^{\text {al }}$, M.H. Hofstadter ${ }^{j}$, R. Holme ${ }^{a m}$, M. Horanyi ${ }^{\text {ah }}$, G. Hospodarsky ${ }^{\text {an }}$, S. Hsu ${ }^{\text {ah }}$, P. Irwin ${ }^{\text {ae }}$, C.M. Jackman ${ }^{\text {ao }}$, O. Karatekin ${ }^{\text {ap }}$, S. Kempf ${ }^{\text {ah }}$, E. Khalisi ${ }^{\text {aq }}$, K. Konstantinidis ${ }^{\text {ar }}$, H. Krüger ${ }^{v}$, W.S. Kurth ${ }^{\text {an }}$, C. Labrianidis ${ }^{\text {as }}$, V. Lainey ${ }^{\text {at }}$, L.L. Lamy ${ }^{\text {t }}$, M. Laneuville ${ }^{\mathrm{au}}$, D. Lucchesi ${ }^{\text {ad }}$, A. Luntzer $^{\mathrm{av}}$, J. MacArthur $^{\mathrm{b}}$, A. Maier ${ }^{\mathrm{aw}}$, A. Masters ${ }^{\text {h,ac }}$, S. McKenna-Lawlor ${ }^{a x}, H_{\text {. Melin }}{ }^{f}$, A. Milillo ${ }^{\text {ad }}$, G. Moragas-Klostermeyer ${ }^{\text {aq }}$,

A. Morschhauser ${ }^{\text {ay }}$, J.I. Moses ${ }^{\text {az }}$, O. Mousis ${ }^{\text {ba }}$, N. Nettelmann ${ }^{\text {af }}$, F.M. Neubauer ${ }^{\text {bb }}$, T. Nordheim ${ }^{\mathrm{a}, \mathrm{b}}$, B. Noyelles ${ }^{\mathrm{bc}}$, G.S. Orton ${ }^{\mathrm{j}}$, M. Owens ${ }^{\mathrm{bd}}$, R. Peron ${ }^{\mathrm{ad}}$, C. Plainaki ${ }^{\mathrm{ad}}{ }^{\text {, }}$, F. Postberg ${ }^{\text {aq }}$, N. Rambaux ${ }^{\text {be,at }}$, K. Retherford ${ }^{\text {ab }}$, S. Reynaud ${ }^{\text {bf }}$, E. Roussos ${ }^{\text {v }}$, C.T. Russell ${ }^{\text {bg }}$, A.M. Rymer ${ }^{\text {s, R. Sallantin }}{ }^{g}$, A. Sánchez-Lavega ${ }^{\text {bh }}$, O. Santolik ${ }^{\text {bi }}$, J. Saur ${ }^{\text {bb }}$, K.M. Sayanagi ${ }^{\text {bj }}$, P. Schenk ${ }^{\text {bk }}$, J. Schubert ${ }^{\text {bl }}$, N. Sergis ${ }^{\text {bm }}$, E.C. Sittler ${ }^{x}$, A. Smith ${ }^{\text {a }}$, F. Spahn ${ }^{\text {bn }}$, R. Srama ${ }^{\text {aq }}$, T. Stallard $^{\text {bo }}$, V. Sterken ${ }^{\text {ag,bq }}$, Z. Sternovsky ${ }^{\text {ah }}$, M. Tiscareno $^{1}$, G. Tobie ${ }^{\text {bp }}$, F. Tosi $^{\text {ad }}$, M. Trieloff ${ }^{\text {al }}$, D. Turrini ${ }^{\text {ad }}$, E.P. Turtle ${ }^{\mathrm{s}}$, S. Vinatier ${ }^{\mathrm{t}}$, R. Wilson ${ }^{\text {ah }}$, P. Zarka ${ }^{\mathrm{t}}$

\footnotetext{
a Mullard Space Science Laboratory, University College London, UK

${ }^{\mathrm{b}}$ The Centre for Planetary Science at UCL/Birkbeck, London, UK

${ }^{\mathrm{c}}$ Department of Physics and Astronomy, University College London, UK

${ }^{\mathrm{d}}$ ESTEC, European Space Agency, The Netherlands

e Queen Mary University of London, UK

${ }^{\mathrm{f}}$ Space Research Centre, University of Leicester, UK

g IRAP, Toulouse, France

${ }^{\mathrm{h}}$ ISAS, JAXA, Japan

i Department of Physics, Lancaster University, UK

${ }^{\mathrm{N}}$ NASA Jet Propulsion Laboratory, USA

${ }^{\mathrm{k}}$ University of Wisconsin-Madison, USA

${ }^{1}$ Cornell, USA

${ }^{\mathrm{m}}$ Univ. Grenoble Alpes, IPAG, F-38000 Grenoble, France

${ }^{\mathrm{n}}$ Rutherford Appleton Laboratory, STFC, UK

${ }^{\circ}$ Technical University, Braunschweig, Germany

${ }^{\mathrm{p}}$ Delft University of Technology, The Netherlands

${ }^{\mathrm{q}}$ Université de Liège, Belgium

${ }^{\mathrm{r}}$ National University of Ireland, Maynooth, Ireland

s Johns Hopkins University Applied Physics Laboratory, USA

${ }^{\mathrm{t}}$ LESIA, L'Observatoire de Paris, France

' $L P C 2 E, C N R S$, Université d'Orléans, Orléans, France

${ }^{\vee}$ Max Planck Institute for Solar System Research, Göttingen, Germany

w ONERA, France

${ }^{x}$ NASA Goddard Space Flight Centre, USA

${ }^{\mathrm{y}}$ ESAC, European Space Agency, The Netherlands

${ }^{\mathrm{z}}$ Department of Physics, University of Athens, Greece

aa University of California, Berkeley, USA

ab Southwest Research Institute, San Antonio, TX, USA

ac Department of Physics, Imperial College London, UK
} 
${ }^{\text {ad }}$ INAF-IAPS Istituto di Astrofisica e Planetologia Spaziali, Rome, Italy

ae Department of Physics, University of Oxford, UK

af University of California Santa Cruz, USA

ag Max Planck Institute for Nuclear Physics, Germany

${ }^{\text {ah }}$ LASP, University of Colorado, USA

ai University of Idaho, Moscow, ID, USA

aj Tel Aviv University, Tel Aviv, Israel

ak LATMOS, France

${ }^{\text {al } H e i d e l b e r g}$ University, Germany

am University of Liverpool, UK

an University of Iowa, USA

ao Department of Physics and Astronomy, University of Southampton, UK

ap Royal Observatory of Belgium, Belgium

aq University of Stuttgart, Germany

ar Universität der Bundeswehr München, Germany

as UTesat-Spacecom GmbH, Germany

at IMCCE-Observatoire de Paris, UMR 8028 du CNRS, UPMC, Université Lille 1, 77 Av. Denfert-Rochereau, 75014 Paris, France

${ }^{\text {au }}$ Institut de Physique du Globe de Paris, France

av University of Vienna, Austria

aw Space Research Institute, Austrian Academy of Science, Austria

ax Space Technology Ireland, National University of Ireland, Ireland

ay DLR, Germany

az Space Science Institute, USA

ba Observatoire de Besançon, France

bb University of Cologne, Germany

bc University of Namur, Belgium

bd University of Reading, UK

be Université Pierre et Marie Curie, UPMC - Paris 06, France

bf Laboratoire Kastler Brossel, CNRS, UMPC, France

bg Institute of Geophysics and Planetary Physics, University of California, Los Angeles, USA

bh University of the Basque Country, Spain

bi Institute of Atmospheric Physics, Prague, Czech Republic

bj Department of Atmospheric and Planetary Sciences, Hampton University, Virginia, USA

bk Lunar and Planetary Institute, University of Arizona, USA

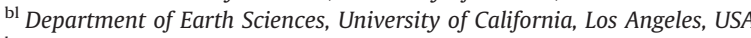

$\mathrm{bm}$ Office for Space Research and Technology, Academy of Athens, Greece

bn University of Potsdam, Germany

bo Department of Physics and Astronomy, University of Leicester, UK

bp LPG, CNRS - Université de Nantes, France

${ }^{b q}$ International Space Science Institute, Bern, Switzerland

br CNRS, IPAG, F-38000 Grenoble, France

\section{A R T I C L E I N F O}

Article history:

Received 17 December 2013

Received in revised form

23 July 2014

Accepted 7 August 2014

Keywords:

Uranus

Magnetosphere

Atmosphere

Natural satellites

Rings

Planetary interior

\begin{abstract}
A B S T R A C T
Giant planets helped to shape the conditions we see in the Solar System today and they account for more than $99 \%$ of the mass of the Sun's planetary system. They can be subdivided into the Ice Giants (Uranus and Neptune) and the Gas Giants (Jupiter and Saturn), which differ from each other in a number of fundamental ways. Uranus, in particular is the most challenging to our understanding of planetary formation and evolution, with its large obliquity, low self-luminosity, highly asymmetrical internal field, and puzzling internal structure. Uranus also has a rich planetary system consisting of a system of inner natural satellites and complex ring system, five major natural icy satellites, a system of irregular moons with varied dynamical histories, and a highly asymmetrical magnetosphere. Voyager 2 is the only spacecraft to have explored Uranus, with a flyby in 1986, and no mission is currently planned to this enigmatic system. However, a mission to the uranian system would open a new window on the origin and evolution of the Solar System and would provide crucial information on a wide variety of physicochemical processes in our Solar System. These have clear implications for understanding exoplanetary systems. In this paper we describe the science case for an orbital mission to Uranus with an atmospheric entry probe to sample the composition and atmospheric physics in Uranus' atmosphere. The characteristics of such an orbiter and a strawman scientific payload are described and we discuss the technical challenges for such a mission. This paper is based on a white paper submitted to the European Space Agency's call for science themes for its large-class mission programme in 2013.
\end{abstract}

(c) 2014 Published by Elsevier Ltd.
* Corresponding author at: Mullard Space Science Laboratory, University College London, UK. Tel.: +441483204150.

E-mail address: c.arridge@ucl.ac.uk (C.S. Arridge).

\section{Introduction}

Giant planets account for more than $99 \%$ of the mass of the Sun's planetary system, and helped to shape the conditions we see in the Solar System today. The Ice Giants (Uranus and Neptune) are fundamentally different from the Gas Giants (Jupiter and Saturn) in a number of ways and Uranus in particular is the most challenging to our understanding of planetary formation and 
evolution (e.g., Lissauer, 2005; Dodson-Robinson and Bodenheimer, 2010). Our Solar System provides the only local laboratory in which we can perform studies that help us to understand the nature of planetary systems in general. The fact that Kepler observations have shown that Uranus/Neptune class planets are a common class of exoplanet (Fressin et al., 2013) makes it all the more timely and compelling to better explore these fascinating systems.

The Ice Giants are fundamentally different from the Gas Giants (Jupiter and Saturn) in a number of ways and Uranus in particular is the most challenging to our understanding of planetary formation and evolution, with its puzzling interior structure, unclear energy balance and internal energy transport mechanisms, and its high obliquity. Yet our exploration of the Ice Giants in our own Solar System remains incomplete, with several fundamental questions unanswered. Voyager 2 remains the only spacecraft to have returned data from the uranian environment, see for example papers in Science 233(4759) from the Voyager 2 Uranus encounter, with an introduction given by Stone and Miner (1986), and the current authoritative book on the Voyager 2 encounter science (Matthews et al., 1991).

A mission to Uranus will provide observations and measurements that are vital for understanding the origin and evolution of Uranus as an Ice Giant planet, answer the fundamental question of why some giant planets become icy and other so gas rich, and provide a missing link between our Solar System and planets around other stars. Observations of Uranus' rings and satellite system will also bring new perspective on the origin of giant planet systems and will help validate the models proposed for the origin and evolution of Jupiter's and Saturn's systems. The cruise phase will also offer the possibility of testing the law of gravitation in a dynamic environment, still poorly probed, and study the outer heliosphere and its connection to the Sun. Such a mission to the uranian system would open a new window on the origin and evolution of the Solar System and directly addresses two of European Space Agency's (ESA) Cosmic Vision themes "What are the conditions for Planet Formation and the Emergence of Life?" and "How Does the Solar System Work?". The fundamental processes occurring within the uranian system confirm that the exploration of Uranus is essential in meeting ESA's Cosmic Vision goals. A mission to Uranus is also highlighted in the NASA Planetary and Heliophysics Decadal Surveys (Squyres et al., 2011; Baker et al., 2013).

In 2013 ESA issued a call for science themes for its large-class (L-class) mission programme. This paper represents the white paper on the scientific case for the exploration of Uranus that was submitted to this call (a compilation of these white papers can be found at http://sci.esa.int/science-e/www/object/doc.cfm?fobjec tid $=52029$ ) and looks forward to future missions. This white paper followed the Uranus Pathfinder mission proposal that was submitted to ESA's medium-class (M-class) mission programme in 2010 which is described in Arridge et al. (2012). In September 2013 a Uranus-focused workshop "Uranus beyond Voyager 2, from recent advances to future missions", was held at the Observatory of Paris (Meudon, France) and was attended by 90 scientists and engineers from 12 countries, interested in the scientific exploration of this unique planetary system (the detailed programme, abstracts and lists of participants is available at http://uranus. sciencesconf.org).

In Section 2 of this paper the science case for a Uranus mission is presented and arranged into three key themes: (1) Uranus as an Ice Giant Planet, (2) An Ice Giant Planetary System, and (3) Uranus' Aeronomy, Aurorae and Highly Asymmetrical Magnetosphere. In addition, a mission to Uranus naturally provides a unique opportunity to study the outer heliosphere, fundamental gravitational physics, and Solar System bodies such as Centaurs near the orbit of
Uranus and so in this paper we also describe the science case associated with a cruise phase in the outer Solar System. The short mission concept that was described in the white paper is presented in Section 3 along with a discussion of the critical enabling technologies.

\section{Scientific case}

\subsection{Uranus as an ice giant planet: The interior and atmosphere of Uranus}

Fig. 1 indicates the bulk composition for various Solar System objects and shows how different the Ice Giants are from the Gas Giants. Jupiter is an $\mathrm{H} / \mathrm{He}$ planet with an ice and rock mass fraction of $4-12 \%$ as inferred from standard interior models (Saumon and Guillot, 2004). Uranus and Neptune seem to consist mostly of ices and rocks, but current observations are only able to provide an upper limit of $85 \%$ on the ice and rock mass fraction (Fortney and Nettelmann, 2010). The self-luminosity of Uranus is the lowest of all the planets in the solar system, suggesting that the interior of Uranus either is not fully convective or that it suffered an early loss of internal heat, perhaps in a giant impact. The internallygenerated magnetic field of Uranus is highly complex and unusual which suggests some fundamental difference between the dynamo in Uranus' interior and those of the Earth and Gas Giants. Understanding the internal structure of Uranus (the nearest Ice Giant) is indispensable for estimating the bulk composition of outer planets, in particular their ice-to-rock ratio. There is currently no interior model for Uranus that agrees with all the observations, representing a significant gap in our understanding

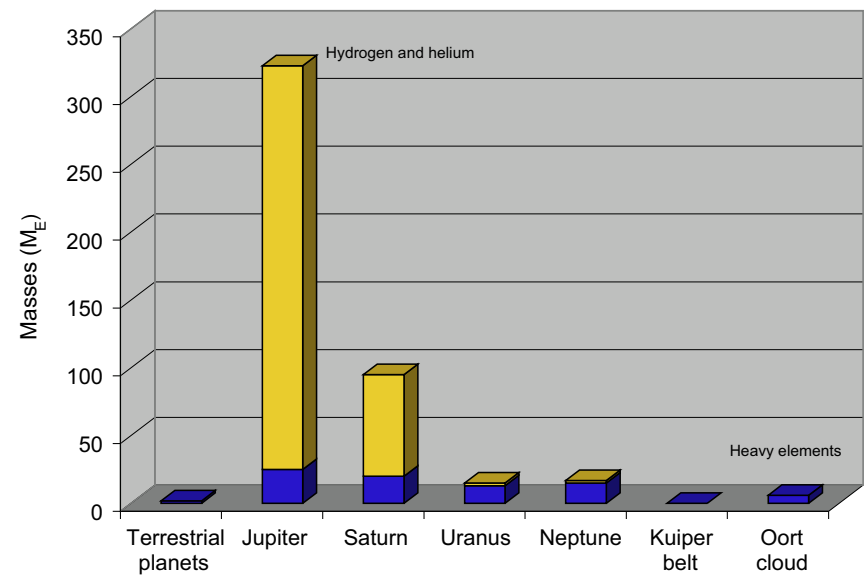

Fig. 1. Composition of various solar system objects, split into hydrogen and helium (yellow) and heavy elements (blue). Modified from Guillot and Gautier (2010). (For interpretation of the references to colour in this figure legend, the reader is referred to the web version of this article.

Table 1

Physical and orbital parameters of Uranus.

\begin{tabular}{ll}
\hline Equatorial radius & $25,559 \mathrm{~km}\left(=1 \mathrm{R}_{\mathrm{U}}\right)$ \\
Mass & $14.5 \mathrm{M}_{\mathrm{E}}$ \\
Sidereal spin period & $17 \mathrm{~h} 12 \mathrm{~min} 36 \mathrm{~s}( \pm 72 \mathrm{~s})$ \\
Obliquity & $-97.77^{\circ}$ \\
Semi-major axis & $19.2 \mathrm{AU}$ \\
Orbital period & $84.3 \mathrm{Earth}$ years \\
Dipole moment & $50 \mathrm{M}_{\mathrm{E}}$ \\
Magnetic field & Highly complex with a surface field up to $110,000 \mathrm{nT}$ \\
Dipole tilt & $-59^{\circ}$ \\
Natural satellites & $27(9$ irregular $)$ \\
\hline
\end{tabular}


of the Solar System. Compared to the Gas Giants, this differing bulk composition and the internal structure reflects the different formation environments and evolution of the Ice Giants relative to the Gas Giants (e.g., Guillot, 2005), providing a window onto the early Solar System. Table 1 lists the gross properties of Uranus. The origin of Uranus' large obliquity is perhaps one of the most outstanding mysteries of our Solar System. A variety of explanations have been invoked, including a giant impact scenario which may also be implicated in Uranus' low luminosity and small heat flux, and tidal interactions (Boué and Laskar, 2010; Morbidelli et al., 2012). Examining the interior structure and composition of Uranus and its natural satellites, and studying the ring system may allow us to unravel the origin of this Solar System mystery.

The composition of Uranus contains clues to the conditions in the protosolar cloud and the locations in which it formed. For instance, a subsolar $\mathrm{C}: \mathrm{O}$ ratio could indicate formation at a distance where water (but not $\mathrm{CH}_{4}$ ) was frozen. The common picture of gaseous planet formation by first forming a $10 \mathrm{M}_{\mathrm{E}}$ core and then accreting a gaseous envelope is challenged by state-ofthe-art interior models, which instead predict rock core masses below $5 \mathrm{M}_{\mathrm{E}}$ (Saumon and Guillot, 2004; Fortney and Nettelmann, 2010). Uranus' inclination and low heat loss may point to another catastrophic event and provides additional important constraints for planetary system formation theory. New observations of Ice Giants are therefore crucial in order to resolve this and achieve Cosmic Vision goals on understanding the formation of planets.

Uranus' atmosphere is unique in our Solar System in that it receives a negligible flux of heat from the deep interior and experiences extremes of seasonal forcing due to the high $98^{\circ}$ obliquity, with each pole spending 42 years in darkness. This unusual balance between internal and radiative heating means that Uranus' unique weather is governed principally by seasonal forcing. Furthermore, the substantial enrichment of some heavy elements (but perhaps not all, $\mathrm{N}$ being strongly depleted in the troposphere) and small envelopes of $\mathrm{H}_{2}-\mathrm{He}$ in the Ice Giants and the cold atmospheric temperatures relative to the Gas Giants yield unique physicochemical conditions. Uranus therefore provides an extreme test of our understanding of planetary atmospheric dynamics; energy and material transport; seasonally varying chemistry and cloud microphysics; structure and vertical coupling throughout giant planet atmospheres. At higher altitudes, the temperature in Uranus' thermosphere is several hundred degrees hotter than can be explained by solar heating (as is also found for Saturn and Jupiter) and remains a fundamental problem in our understanding of giant planet upper atmospheres in general (e.g., Herbert et al., 1987). Even though Earth-based observations of Uranus (Infrared Space Observatory, Spitzer, Herschel, groundbased) have improved dramatically in the decades since Voyager 2, many questions about this very poorly explored region of our Solar System remain unanswered. Certain spectral regions, particularly those obscured by telluric water vapour, are inaccessible from the ground. The overarching atmospheric science objective is to explore the fundamental differences in origin, meteorology and chemistry between the Ice and Gas Giants; to reveal the underlying mechanisms responsible for Uranus' unique conditions.

\subsubsection{What is the internal structure and composition of Uranus}

At present there is no Uranus interior model that is consistent with all of the physical constraints, such as Uranus' gravity field, luminosity, magnetic field, and realistic ice-to-rock ratio. Fig. 2 illustrates a model that is consistent with the gravity and magnetic field data but not with the luminosity of the planet. Uranus and Neptune are known to have substantial elemental enrichments in carbon and deuterium (Owen and Encrenaz, 2006; Feuchtgruber et al., 2013), but abundances of other simple elements (N, S and O),

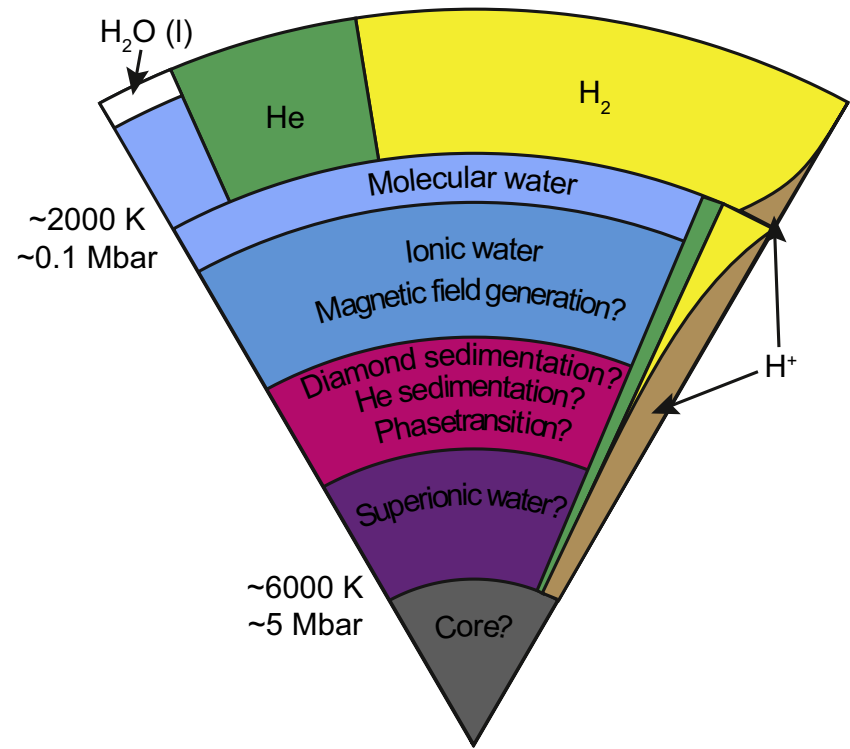

Fig. 2. Model of Uranus' interior.

their isotopic ratios $\left({ }^{12} \mathrm{C} /{ }^{13} \mathrm{C},{ }^{14} \mathrm{~N} /{ }^{15} \mathrm{~N},{ }^{16} \mathrm{O} /{ }^{17} \mathrm{O}\right)$ and the noble gases ( $\mathrm{He}, \mathrm{Ne}, \mathrm{Ar}, \mathrm{Xe}, \mathrm{Kr}$ ) have never been adequately constrained. Nevertheless, Uranus' bulk atmospheric composition provides a key diagnostic of planetary formation models. To develop improved models of Uranus' interior, better compositional data must be obtained (Helled et al., 2010).

The mass of the core also places constraints on planetary formation models. For example, if $\mathrm{H} / \mathrm{He}$ is mixed into the deep interior with only a small central core this could suggest gas accretion onto a low-mass proto-planetary core, or efficient vertical mixing, or inclusion of disk-gas into the building planetesimals, rather than accretion onto a large ice-rock core of $\sim 10 \mathrm{M}_{\mathrm{E}}$. Furthermore, the predicted large size of Uranus' core relative to the $\mathrm{H}_{2}-\mathrm{He}$ envelope may make Uranus our best opportunity for studying the elemental composition and thermochemistry of the outer solar nebula at the earliest stages of planetary formation. Measurements of Uranus' bulk atmospheric composition, luminosity, magnetic and gravity fields, and normal-mode oscillations will place new constraints on Uranus' interior and on the origins and evolution of Uranus. The gravity field can be measured both by radio science and by observing the precession of Uranus' ten dense narrow elliptical rings (Jacobson et al., 1992; Jacobson, 1998, 2007). Magnetic field measurements can be used to assess the structure of the dynamo region. Measurement of noble gas abundances and isotopic ratios can be obtained with a shallow (1 bar) entry probe, whilst some isotopic ratios can be determined by remote sensing. A deep ( $>5$ bar) atmospheric entry probe would be able to resolve the question of whether the $S / N$ ratio is enhanced above solar abundance. Giant-planet seismology, building upon the mature fields of helio- and astro-seismology, will revolutionise our ability to probe the interior structure and atmospheric dynamics of giant planets.

Improved knowledge of the composition and interior structure of Uranus will also provide deeper insight into the processes that remixed material in the protoplanetary disk, caused for example by the formation of Jupiter (Safronov, 1972; Turrini et al., 2011) or due to extensive primordial migration of the giant planets (Walsh et al., 2011).

\subsubsection{Why does Uranus emit very little heat?}

Planets are warm inside and cool down as they age. Gravitational energy from material accretion was converted to intrinsic, 
thermal energy during formation and is steadily radiated away through their tenuous atmospheres as they age. Voyager measurements suggest that Uranus' evolution produced a planet with negligible self-luminosity, smaller than any other planet in our Solar System (Pearl et al., 1990). Thermal evolution models probe the energy reservoir of a planet by predicting its intrinsic luminosity. Such models reproduce the observed luminosity of Jupiter and Neptune after $4.56 \mathrm{Gyr}$ of cooling, independent of detailed assumptions about their atmosphere, albedo, and solar irradiation. The same models, however underestimate it for Saturn and overestimate it for Uranus. Indeed, Uranus's atmosphere appears so cold (its intrinsic luminosity so low) that, according to standard thermal evolution theory, Uranus should be more than 3 Gyr older that it is. However, the uncertainties on the Voyagerdetermined energy balance are large enough to substantially reduce that discrepancy. In particular, as the observational uncertainty (Pearl et al., 1990) in the albedo and the effective temperature (derived from the brightness temperature) are significant, Uranus could as well cool down adiabatically, just as Neptune, if its real heat loss is close to the observed upper limit.

The small self-luminosity, combined with the sluggish appearance of the atmosphere as viewed by Voyager, suggests that the interior of Uranus is either (a) not fully convective or that (b) it suffered an early loss of internal heat. Case (b) would suggest that the interior is colder than in the adiabatic case, with crystalline water deep inside (Hubbard et al., 1995). This points to a catastrophic event in Uranus' early history that shocked the matter and led to a rapid energy loss. In case (a) we would expect the interior to be warmer, with water plasma (e.g., Redmer et al., 2011) implying large-scale inhomogeneities, possibly caused by immiscibility of abundant constituents such as helium and carbon or upward mixing of core material, that inhibit efficient heat transport. However, during the last decade ground-based observations have revealed the appearance of convective cloud features, typically at mid-lattiudes, suggesting localised convective regions of adiabatic thermal gradients in the deep troposphere (Sromovsky et al., 2007; de Pater et al., 2011). Vertical transport of energy and material seems to occur only in localised regions on this enigmatic planet. In fact, the inferred size of a non-convective internal region depends sensitively on the imposed intrinsic heat flux value: a mostly stable interior is predicted if the heat flux is close to zero, but a fully convective interior is possible, as for Neptune, should the upper limit of the observed heat flux value prove true.

In order to better constrain Uranus' internal heat flux (derived from the measured albedo and brightness temperature) tighter observational constraints of these quantities are necessary. These inferences come from a single measurement from the Voyager flyby, at a single point in Uranus' seasonal cycle. Thus the balance between Uranus' emission and absorption may be seasonally variable, and new global measurements of reflected solar and emitted infrared radiation are required to assess the presence or absence of an internal heat source, and its importance as driving mechanisms for Uranus' meteorological activity. Atmospheric properties and profiles, measured by an atmospheric entry probe using a combination of radio science, an on-board accelerometer and a nephelometer, may also shed light on heat transport in the atmosphere.

\subsubsection{What is the configuration and origin of Uranus' highly asymmetrical internal magnetic field?}

Understanding the configuration of Uranus' internal magnetic field is essential for correctly interpreting the configuration of the magnetosphere, its interaction with the rings, moons and solar wind, and for understanding how dynamo processes in the interior of Uranus generate the field. In contrast to the magnetic fields of Earth, Mercury, Jupiter and Saturn, which are dominated by a dipole nearly co-aligned with the rotation axis, those of Uranus and Neptune are characterised by a large offset and tilt between the dipole and spin axes with strong quadrupole and octupole contributions to the internal magnetic field. The magnetic field data from Voyager 2 are sufficient to crudely constrain the internal field of Uranus, but more complex and (currently) poorly constrained models are required to fit the data (Holme and Bloxham, 1996). At the planetary surface the magnetic dipole, quadruople and octupole components of the total internal field are of comparable strength, but at the top of the dynamo region $\left(\sim 0.75 \mathrm{R}_{\mathrm{U}}\right)$ the latter two dominate. Fig. 3 illustrates the highly asymmetrical nature of Uranus' internal magnetic field, using the model of Herbert (2009) compared with Saturn's highly symmetrical internal field, using the model of Davis and Smith (1990).

A variety of competing numerical dynamo models (e.g., Stanley and Bloxham, 2004, 2006; Soderlund et al., 2013) have been developed which can explain these fields but new magnetic field measurements are required to allow us to determine which is the closest to reality. The field is also expected to have undergone secular changes since the Voyager 2 epoch (Christensen and Tilgner, 2004). Magnetic field measurements at a variety of planetocentric latitudes and longitudes will provide a wealth of

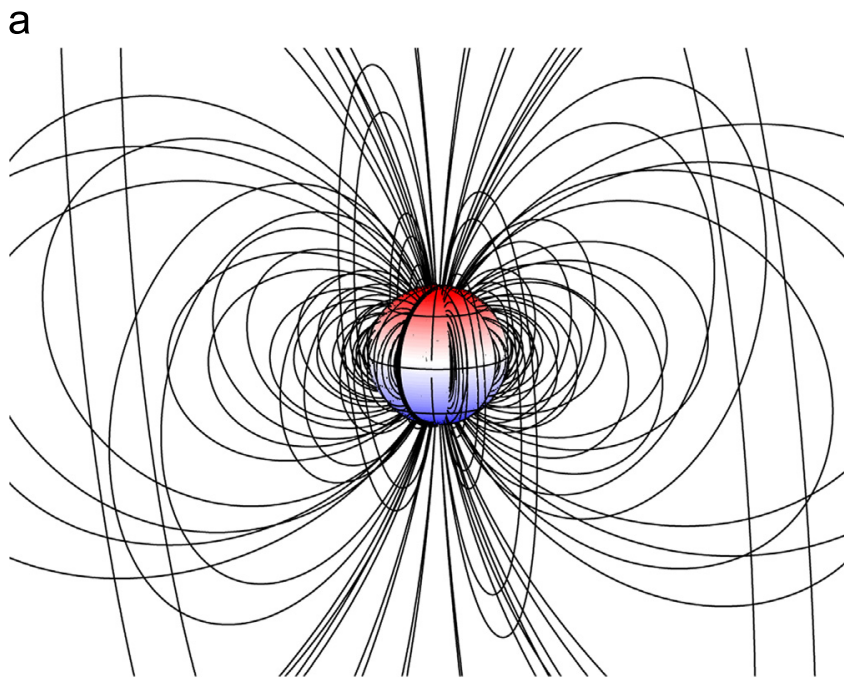

b

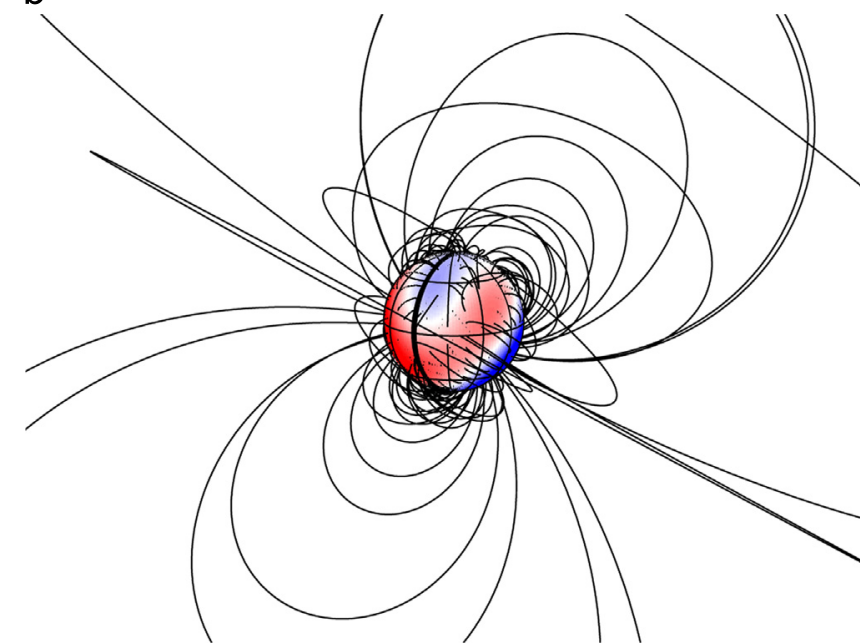

Fig. 3. Comparison of Uranus' highly asymmetrical field with Saturn's symmetrical field using the models of Herbert (2009) and Davis and Smith (1990), respectively. The colour scale indicates the magnitude of the radial field at the 1 bar level. (a) Saturn field (Davis and Smith, 1990) and (b) Uranus field (Herbert, 2009). 
data from which to test these competing models. This will lead to significant changes in our understanding of field generation in Ice Giant planets and of planetary magnetic field generation in general. Models of the internal field can also be greatly improved by the use of auroral images which provide additional highlatitude constraints. Herbert (2009) combined the Voyager observations of the internal field and assumed magnetically conjugate southern and northern UV auroral emissions to derive such a higher order model. Better-quality images of auroral emissions than are possible from Earth (e.g., Lamy et al., 2012) are paramount for improving the accuracy of the planetary field model.

\subsubsection{What is the rotation rate of Uranus' interior?}

A correct interpretation of the internal structure of Uranus relies on an accurate knowledge of the internal rotation rate of the planet (Nettelmann et al., 2013). Modelling of Uranus' internal magnetic field, and observations of radio emissions (Uranian Kilometric Radiation (UKR)) and atmospheric motions all provide independent estimates of the rotation rate of the planet, although not always from the same region of the planet. Analyses of Voyager 2 data have yielded three estimates of the rotation rate of Uranus, from $17 \mathrm{~h} 12 \mathrm{~min} 36 \mathrm{~s}$ ( $\pm 72 \mathrm{~s}$ ) (Herbert, 2009) to $17 \mathrm{~h}$ 17 min 24 s ( \pm 36 s) (e.g., Ness et al., 1991). New measurements of Uranus' magnetic field and UKR will enable us to significantly improve the accuracy on the determination of the planetary period (to a few parts in $10^{-5}$ ), and check if second order effects (e.g., Saturn displays different radio periods in both magnetic hemispheres, each varying with time) are present.

\subsubsection{How is Uranus' atmospheric structure and composition influenced by its unique seasons?}

The potential absence of an internal heat source renders Uranus' weather unique among the giant planets. Neptune, with its powerful self-luminosity, provides an important counterexample of a convectively-active weather layer. The extreme $98^{\circ}$ obliquity of Uranus subjects the atmosphere to extremes of seasonal forcing, with each pole spending decades in darkness. Despite the bland visible appearance of Uranus from Voyager, recent ground-based observations (e.g., Sromovsky et al., 2007, 2009, 2014; de Pater et al., 2011; Fry et al., 2012) have shown the planet to be more dynamically active than previously thought (Fig. 4).

Bright poles seen in 1.3-cm images from the Very Large Array (VLA) show that the polar regions of the deep troposphere are depleted in absorbers relative to the equator, thus indicating large- scale atmospheric motions (Hofstadter et al., 2006). The same pattern is seen in the $\mathrm{CH}_{4}$ distribution at higher altitudes (Karkoschka and Tomasko, 2009) (Fig. 5). Seasonal changes in clouds and dynamics have also been observed: in 1986, the sunlit South Pole appeared bright due to a polar 'cap' of stratospheric aerosols. The bright South Pole diminished over the ensuing years, and became a faint polar band of brighter material, while a new collar of bright material became visible in the northern springtime hemisphere. High resolution ground-based observations in 2012 (Fig. 4) reveal what may be convective clouds of $\mathrm{CH}_{4}$, which may eventually form a polar hood as was seen in the southern polar regions during the Voyager flyby (e.g., Atreya et al., 1991). All of these are indicative of the meridional circulation, which on this highly seasonally driven planet is likely to be unique, but also instructive about how planets work under more general obliquity/ insolation conditions. The long temporal baseline of high spatial resolution atmospheric observations will allow us to study the nature, frequency, distribution and morphology of discrete cloud activity (e.g., storms, vortices). In particular, we aim to understand the origin, lifecycle and drift rates of Uranus' dark spots and associated bright clouds (large anticyclonic vortices, e.g., Hammel et al., 2006), for a direct comparison with the lifecycles observed on Neptune. Finally, the relative importance of wave activity versus moist convection in vertical mixing could be uniquely tested on Uranus, given the anticipated low levels of convective activity.

\subsubsection{What processes shape atmospheric chemistry and cloud formation on an ice giant?}

Reflected sunlight observations can be used to identify the composition and distribution of Uranus' main condensation cloud decks. The brightest white features are thought to be caused by ices of $\mathrm{CH}_{4}$, overlying a putative cloud of $\mathrm{NH}_{4} \mathrm{SH}$ or $\mathrm{H}_{2} \mathrm{~S}$, but probably not $\mathrm{NH}_{3}$ (de Pater et al., 1991), with a deep cloud of water hypothesised at much higher pressures. Thin photochemical haze layers may exist above the condensate clouds in the upper troposphere and stratosphere, leading to oscillations in the temperature profiles due to localised radiative heating. Indeed, stratospheric hazes of small particles (likely to be condensed hydrocarbons) were observed in high-phase angle imaging from Voyager 2 (Rages et al., 1991), a geometry that can only be provided by a visiting spacecraft. These condensed hydrocarbons may sediment downwards into the troposphere, serving as cloud condensation nuclei or as coatings for existing particles, complicating our capabilities for uniquely identifying the composition of the cloud decks.

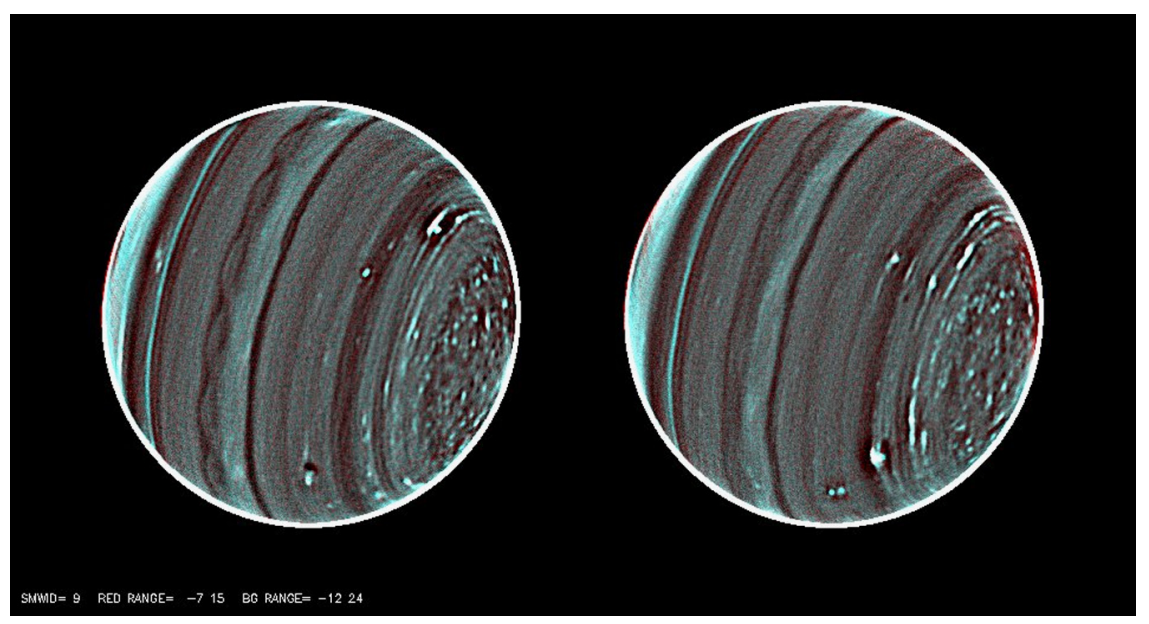

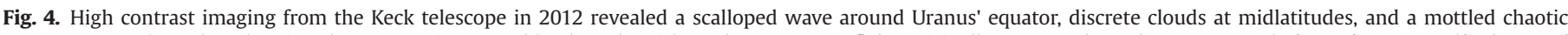

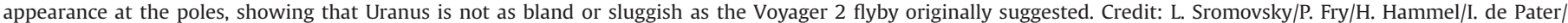
University of Wisconsin-Madison/WM Keck Telescope. 


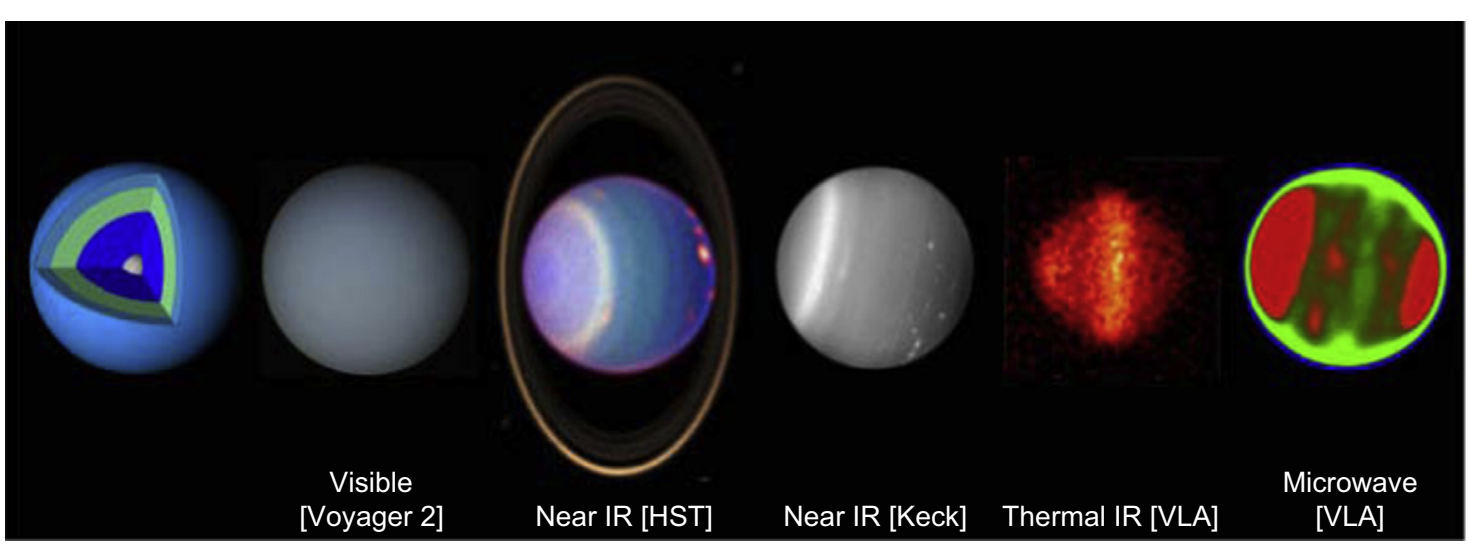

Fig. 5. Images of Uranus in a variety of wavelengths. After Arridge et al. (2012).

The optical properties and spatial distributions of these tropospheric and stratospheric hazes are poorly known, but they may contribute significantly to the radiative heating of the upper atmosphere, and thus our understanding of atmospheric circulation in Uranus' stably-stratified atmosphere.

Below the clouds, the atmospheric composition is poorly known. The altitude of the deep $\mathrm{H}_{2} \mathrm{O}$ condensation cloud is poorly understood because the bulk water abundance may be enhanced by 10-30 times the solar abundance (de Pater and Lissauer, 2010). The $\mathrm{H}_{2} \mathrm{O}$ cloud may exist over extended pressure ranges beneath 50-80 bar, and may even merge with a region of super-critical $\mathrm{H}_{2} \mathrm{O}$ in Uranus' interior. It is not clear what chemical gradients are responsible for the emergence of dark spots (anti-cyclones) and associated bright orographic clouds. Above the clouds, the Infrared Space Observatory (ISO, 1995-1998) and Spitzer Space Telescope (2003-Present) showed that stratospheric chemistry initiated by UV-driven photolysis of $\mathrm{CH}_{4}$ powers a rich photochemistry, resulting in a soup of hydrocarbons in the upper atmosphere (Moses et al., 2005). This hydrocarbon chemistry differs from the other giant planets, as the sluggish vertical mixing means that $\mathrm{CH}_{4}$ is not transported to such high altitudes, so that hydrocarbon photochemistry operates in a very different regime (i.e., higher pressures) than on the other giants. Furthermore, ISO and Herschel (2009-2013) observed oxygenated species in the high atmosphere, potentially due to infalling dust and comets (Feuchtgruber et al., 1997; Cavalié et al., 2014). It is important to search for previously unidentified or unmapped stratospheric species $\left(\mathrm{CO}, \mathrm{HCN}, \mathrm{CO}_{2}\right.$, etc.) such as those related to coupling between the neutral atmosphere and the uranian ring/satellite system.

Remote sounding observations are required to place constraints on Uranus' bulk inventory, vertical distribution, composition, and optical properties of Uranus' clouds and hazes. A deep ( $>5$ bar) atmospheric entry probe would enable the measurement of bulk $\mathrm{CH}_{4}$ and $\mathrm{H}_{2} \mathrm{~S}$ abundances, as well as the abundances of key noble gases and isotopic ratios to understand the origin of this ice giant.

\subsubsection{What processes govern upper atmospheric structure?}

The thermosphere and ionosphere form a crucial transition region between interplanetary space and the planet itself. Powerful currents, generated by electric fields imposed by the magnetosphere, can result in large energy inputs to the upper atmosphere; the energy from these sources may be tens to hundreds of times greater than that due to the absorption of solar (extreme ultraviolet (EUV)) radiation. The unique orientations of Uranus' magnetic dipole and spin axis combined with strong seasonal driving produce a highly time-dependent and complex interaction between the solar wind, magnetosphere, ionosphere and thermosphere. Therefore, this system provides a unique opportunity to understand how insolation and particle precipitation from the solar wind magnetosphere contribute to the energy balance in the upper atmosphere. These processes are suspected to be involved in maintaining a temperature several hundred Kelvin hotter than can be explained by solar heating alone. This requires additional heating and the apparent partial seasonal control (Melin et al., 2011,2013 ) suggests that this is strongly modulated by the way in which varying magnetospheric configurations couple with the upper atmosphere to produce time-variable fields and currents.

Mapping temperatures, electron densities, and the distributions of ions and molecules in the ionosphere and thermosphere using UV and IR remote sensing (in concert with in situ magnetospheric fields and particles measurements, Section 2.3) will permit an unravelling of the thermospheric heating problem and will provide evidence for auroral activity in response to varying solar activity.

\subsection{An ice giant planetary system: rings and natural satellites}

Uranus has a rich planetary system of both dusty and dense narrow rings, and regular and irregular natural satellites. This unique example of a planetary system holds an important key to help us unravel the origin and evolution of the Solar System. Fig. 6 illustrates some of the main features of rings and natural satellites over a wide range of radial distances and Fig. 7 shows a zoom of the inner region from Uranus to Miranda, the innermost of the five major moons. From this figure one can see the dense packing of the uranian ring and inner satellite system. Ground-based observations have found changes in the rings and satellites since the Voyager 2 flyby, indicating that fundamental instabilities in the coupled ring-moon system are of clear importance for understanding the evolution of planetary systems (de Pater et al., 2007). Fig. 8 shows Voyager's single high-phase image of Uranus' ring system, revealing a plethora of dust structures. More recent observations have revealed an outer ring system (Showalter and Lissauer, 2006; de Pater et al., 2006b, 2013). However, yet the lack of a near-infrared spectrometer on Voyager 2 means that the composition of the rings is almost entirely unknown. It is clear from the albedo that the ring particle surfaces, and possibly the particles themselves, are very different from those in Saturn's ring and must include a non-water-ice component.

The five largest moons of Uranus (Miranda, Ariel, Umbriel, Titania, Oberon - see Fig. 9) are comparable in sizes and orbital configurations to the medium-sized moons of Saturn. They are, however, characterised by larger mean densities, about $1500 \mathrm{~kg} \mathrm{~m}^{-3}$ on average, and by different insolation patterns, with their poles directed towards the Sun during solstice, owing to the large axial tilt of the planet. Oberon lies outside of the magnetosphere (depending on season, during solstice it spends 


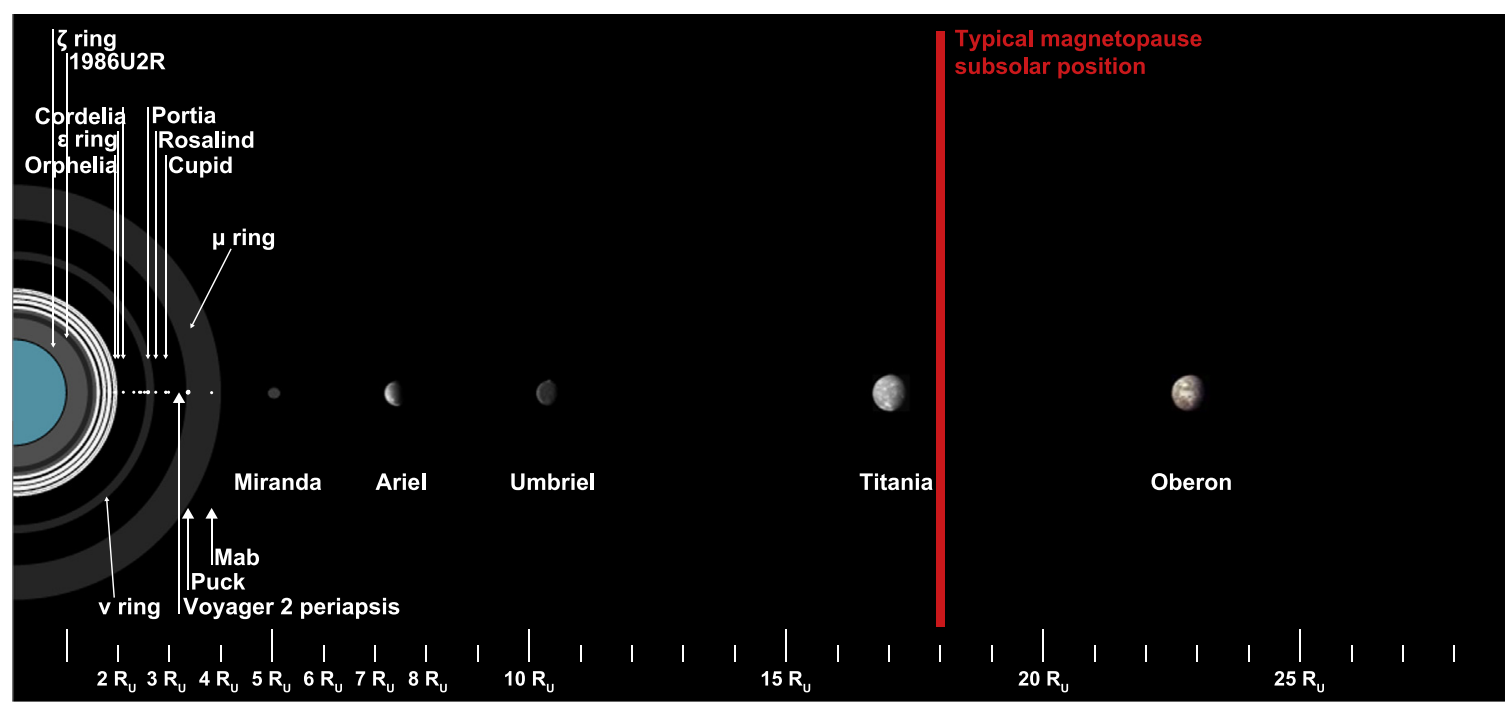

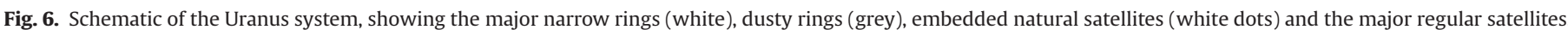

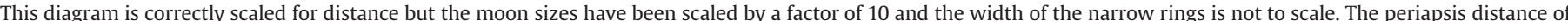

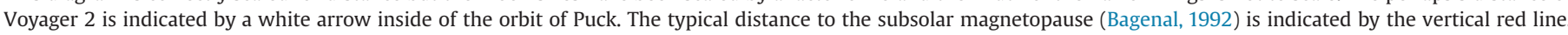

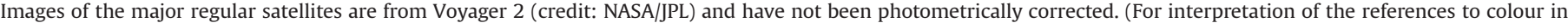
this figure legend, the reader is referred to the web version of this article.)

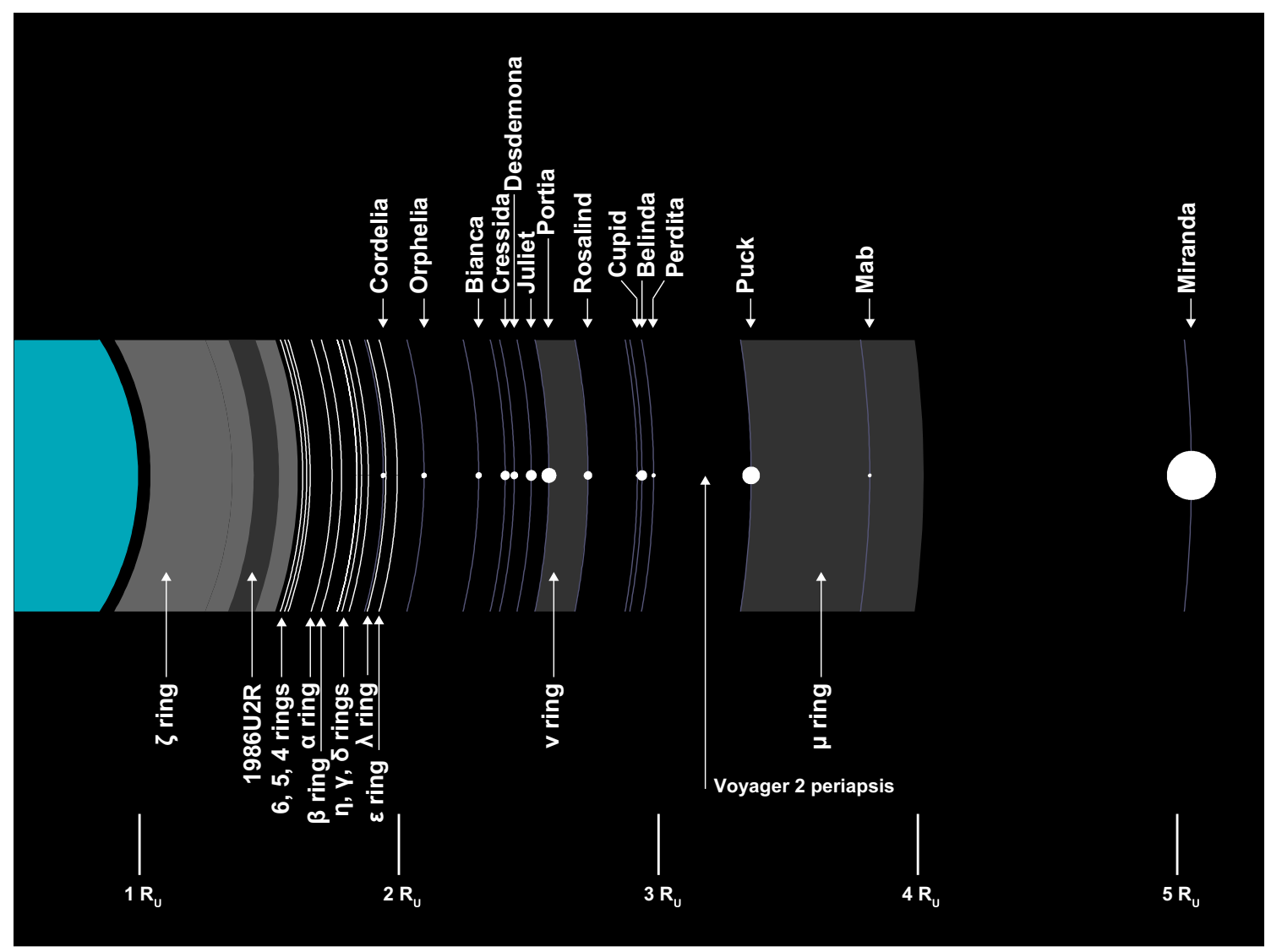

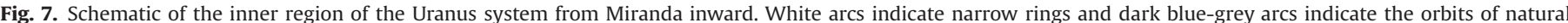

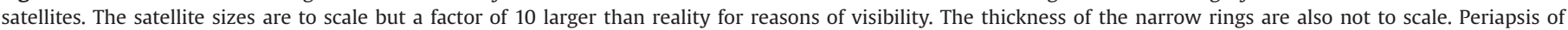

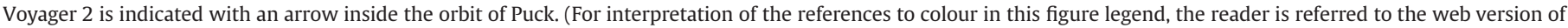
this article.)

periods in the magnetotail), and Titania is sometimes outside the magnetosphere depending on the upstream solar wind conditions (Fig. 6), but Miranda, Ariel and Umbriel orbit within the magnetosphere and hence space weathering should have modified their surface properties, causing particles to be ejected from their surfaces. The observations performed during the flyby of Voyager 2 revealed surprising amounts of geological activity on these moons, possibly involving cryovolcanic processes. Finally, the uranian 
system is host to a set of irregular moons with evidence for dynamical groupings that may hold keys to understanding the evolution of Uranus, in particular the great collision hypothesis for the obliquity of Uranus.

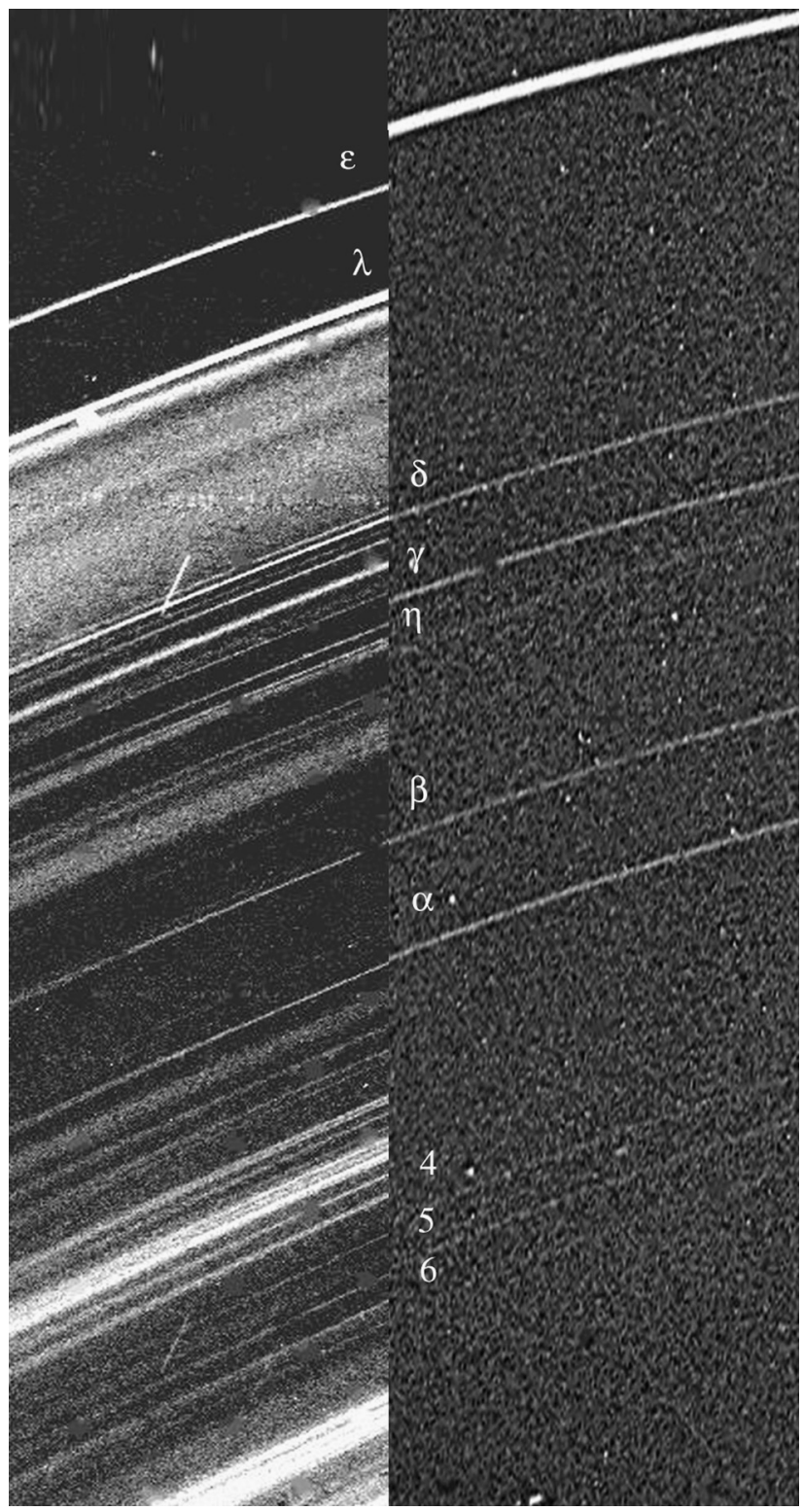

Fig. 8. Composite image of Uranus' main rings in forward-scattered (left) and backscattered (right) light. The left-hand image is the only image of Uranus' rings taken at a high phase angle (by Voyager 2 after closest-approach). These images show that the dense main rings are interleaved with a network of dust structures and the details of how these structures work is largely unknown. Credit: NASA/JPL.
The study of the moons and rings of Uranus - in particular their composition and dynamical stability, their subsurface and deep interior structure, and their geological history and evolution and how that relates to their formation - are important parts of ESA's Cosmic Vision goal for understanding how the Solar System works. The possibility that Uranus' irregular satellites are captured Centaurs or comets can also contribute to understanding small primitive bodies and may provide lessons for our understanding of the origin of life in the Solar System, particularly since objects exposed to the solar wind are subjected to very different space weathering processes than those protected from the solar wind (e.g., Fig. 6).

\subsubsection{What is the composition of the uranian rings?}

The composition of the uranian rings is almost entirely unknown, as Voyager 2 did not carry an infrared spectrometer capable of detecting the rings. However, it is clear from their low albedo that at least the surfaces of the ring particles are very different from those in Saturn's rings, and must have a significant non-water-ice component. The particle-size distribution of Uranus' main rings is also mysterious, where the main rings contain particles between $10 \mathrm{~cm}$ and $10 \mathrm{~m}$, with a surprising lack of cm-size particles detected by the Voyager 2 radio occultation (French et al., 1991). The ring system has also changed significantly since the Voyager flyby in ways we do not understand (Showalter and Lissauer, 2006) and new rings and satellite components have been discovered. These need to be characterised at close range in order to understand how their rapid evolution fits into various paradigms of Solar System evolution.

A Uranus orbiter will enable high-resolution near-infrared and visible observations of the rings and small moons which will constitute a significant advance in our understanding of the evolution of the uranian system and will provide constraints on planetary evolution models. Observations of the narrow rings are needed to unravel the dynamics of their confinement and to confirm theories of self-maintenance and of shepherding by moons, which are relevant to other disk systems including protoplanetary disks. Mapping the spatial variations of both composition and particle size will clarify phenomena such as contamination and material transport within the system. Stellar, solar and radio occultations will enable the determination of the ice-fraction and size distribution of ring particles. A dust detector can directly determine from in situ measurements the number densities as well as the speed and size-distributions of dusty ring material. Moreover, a chemical analyser subsystem can provide unique information on the composition of these grains, bearing the possibility to constrain isotopic ratios of the constituents (Briois et al., 2013). Because larger ring particles and the uranian satellites are the main sources of the dust, dust measurements give direct information on the composition of these bodies. Also of interest are the rings' interaction with Uranus' extended exosphere and their accretion/ disruption interplay with the nearby retinue of small moons.

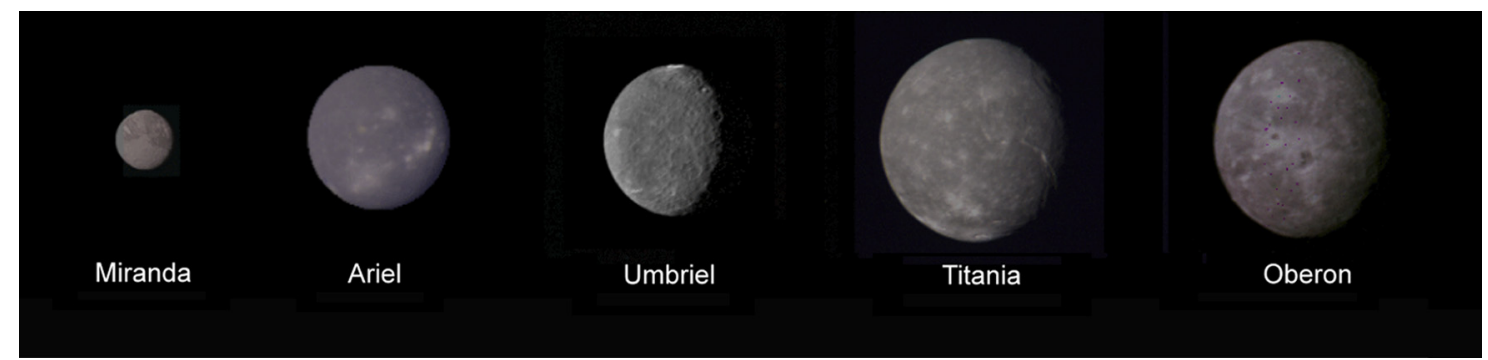

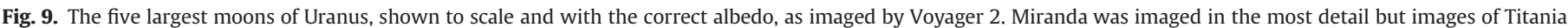
and Oberon were not of a sufficiently high resolution to resolve details of tectonic structures. Credit: Paul Schenk/NASA/JPL. 


\subsubsection{How do dense rings behave dynamically?}

The main rings are eccentric and inclined and generally bounded by sharp edges - see reviews by Elliot and Nicholson (1984) and French et al. (1991). Although theories exist regarding these characteristics, including resonant interactions, "shepherding" by nearby satellites, and self-maintenance, the mechanisms are far from understood. Our understanding of these mechanisms is highly relevant to other disc systems, including protoplanetary and debris discs. Existing data give preliminary hints that self-gravity wakes and spiral density waves, which are important diagnostics as well as driving phenomena in Saturn's rings (e.g., Cuzzi et al., 2010), also exist in at least some parts of Uranus' rings, but much more detailed observation is needed to characterise them.

The rings of Uranus are the best natural laboratory for investigating the dynamics of dense narrow rings, an important complement to the dense broad disk exemplified by Saturn's rings, and diffusive rings at Jupiter and Neptune (Tiscareno, 2013). These observations will undoubtedly reveal many new structures and periodicities, and possibly new moons that play important roles in ring confinement. Rings can also shed light on the planet's gravitational and magnetic fields as well as the influx of interplanetary meteoroids (e.g., Hedman and Nicholson, 2013). High-resolution images of the rings from a number of orbits and phase angles are needed in order to unravel their dynamics.

\subsubsection{How do Uranus' dusty rings work?}

The Cassini mission has taught us that dusty rings are shaped by solar radiation forces, which depend on particle properties (size, albedo, etc.), as well as by the gravitational influence of satellites. Thus, a study of the dynamical structure of dusty rings will unveil much about the particles' currently unknown material properties.

The post-Voyager discovery of the $v$ ring is especially intriguing, as this dusty ring lies between the orbits of two closelypacked satellites, but does not itself have any apparent source (Showalter and Lissauer, 2006). It is quite possible that the $v$ ring is the remains of a moon that was disrupted by a collision fairly recently. The innermost dusty $\zeta$ ring appears to have moved several thousand kilometres outward between the Voyager 2 flyby and recent Earth-based observations (de Pater et al., 2007), but this changing ring has not been studied closely. Ring particles could be lost to the planet by the drag force from the extended exosphere of Uranus (Broadfoot et al., 1986) and may lead to similar effects as the 'ring rain' at Saturn (O'Donoghue et al., 2013). Finally, Voyager's single high-phase image of the rings revealed a plethora of otherwise unknown dust structures (Murray and Thompson, 1990). The bright bands and gaps in this dusty region are difficult to reconcile with conventional theories.

High-resolution images of these dusty rings will allow us to determine their structure and evolution. Detailed observations may reveal one or more large source objects for this dusty region with possible evidence of accretion among them. In situ detection with a dust detector, together with radio and plasma wave observations, would permit a direct measurement of the local dust density, possibly leading to the discovery of new dust populations (Kempf et al., 2005) and interactions with the magnetosphere (Hsu et al., 2011). A dust detector can also provide information on the size-distribution (Spahn et al., 2006) and the composition of grains (Postberg et al., 2011), as well as on their charge state, which might be key (Horányi, 1996) to understand the individual (Horányi et al., 1992) and collective Hedman et al. (2010) dynamics of micron-sized particles. Such in situ measurements have the potential to reveal the mechanisms behind the rapid evolution of the uranian dust rings seen in ground-based data (de Pater et al., 2007) and the intriguing similarities to other ring systems (de Pater et al., 2006a).

\subsubsection{How do the rings and inner satellites interact?}

The inner moons of Uranus comprise the most densely-packed known satellite system, as can be seen in Fig. 7, with 13 knownobjects on orbits ranging from 49,770 to $97,700 \mathrm{~km}$ (Cordelia to Mab) from the planet's centre. This crowded system appears to be subject to mutual collisions on timescales as short as $\sim 10^{6} \mathrm{yr}$ (Duncan and Lissauer, 1997; Showalter and Lissauer, 2006; French and Showalter, 2012), and several moons show measurable orbital changes within a decade or less, raising important questions regarding the origin, evolution, and long-term stability of the Uranus system. Lying immediately exterior to Uranus' main ring system, but outside the "Roche limit" so that collisional products are able to re-accrete into new moons, these uranian inner satellites both interact with the rings (as well as with each other) and comprise a parallel system, a natural laboratory in which the effects of collisional disruption and re-accretion can be studied. The moon Mab lies at the centre of the $\mu$ ring, which shares with Saturn's E ring the unusual characteristic of a blue colour likely due to a preponderance of monodisperse small particles (de Pater et al., 2006b). However, while Enceladus creates the E ring by means of a fine spray of water crystals escaping from geysers, Mab seems much too small ( $\sim 50 \mathrm{~km}$ across) to plausibly sustain any internal activity; it is, however, important to note that the same was formerly said of Enceladus. Mab also exhibits large unexplained deviations in its orbit (Showalter et al., 2008). Close observations of the surface of Mab, as well as its orbit and its interaction with the $\mu$ ring, are certain to yield significant discoveries on the evolution of coupled ring-satellite systems. Astrometric imaging of the uranian inner moons would significantly contribute to understanding this system, identifying resonant and chaotic interactions that can explain its current workings and past history.

\subsubsection{What is the origin of the ring/inner satellite system?}

The close packing of Uranus' small moons and its ring system has suggested that there could be a genetic link between the two. Colwell and Esposito (1993) have suggested that Uranus' rings may be the debris of moons destroyed by the meteroid bombardment over the age of the Solar System. The giant impact theory for Uranus' large obliquity also provides a mechanism for producing the rings from a disruption of the original satellite system (Coradini et al., 2010). More recently it has been suggested that tides themselves may destroy moons and create the rings (Leinhardt et al., 2012). These scenarios are similar to recent suggestions that the satellite systems of Saturn, Uranus and Neptune may have resulted from ring evolution (Crida and Charnoz, 2012). These scenarios would imply the existence of a cycle of material between rings and moons. Since Uranus' ring/ moon system evolves on timescales as short as decades, in situ tracking of this evolution would be a formidable opportunity to study this cycle, which may be at work also for Neptune and Saturn, but on longer time-scales for these systems. By leading a comparative study of spectral characteristics of the rings and moons, we may unveil the origin of both the satellites and rings by inferring whether they are made of the same material or not.

\subsubsection{What is the composition of the uranian moons?}

The five major satellites of Uranus (Miranda, Ariel, Umbriel, Titania, and Oberon) are comparable in orbital configuration and sizes to the medium-sized icy moons of Saturn, but with markedly higher mean densities ( $1500 \mathrm{~kg} \mathrm{~m}^{-3}$ on average). Fig. 9 shows photometrically correct and equal-scale images of these five 
moons. The albedos of the five major satellites of Uranus, varying between 0.21 and 0.39 , are considerably lower than those of Saturn's moons, except Phoebe and the dark hemisphere of Iapetus. This reveals that water ice, which dominates their surfaces, is mixed in varying proportions to other non-ice, visually dark and spectrally bland, material that is possibly carbonaceous in origin (Brown and Cruikshank, 1983). Carbon dioxide has been detected from telescopic observations on Ariel, Umbriel and Titania, but has not been detected on the furthest regular Uranian satellite, Oberon (Grundy et al., 2006). The detected $\mathrm{CO}_{2}$ ice appears to be concentrated on the trailing hemispheres of these satellites, and it decreases in abundance with increasing semimajor axis (Grundy et al., 2006), as opposed to what is observed in the Saturn system.

Due to the absence of a near infrared spectrometer in the payload of Voyager 2, no detailed information is available on the surface chemistry of the icy moons. Just to give a few examples, there is no indication about the chemistry of the structural provinces identified on the surfaces of Titania and Oberon, exhibiting different albedos and different crater density that reveal different ages. Similarly unknown is the nature of dark material (perhaps rich in organics) that fills the floors of major impact craters on Oberon, as well as the composition of the annulus of bright material that is enclosed in the large crater Wunda on Umbriel. The chemical nature of the flows of viscous material observed on Ariel and Titania is also unknown, while a clear indication of the presence of ammonia hydrate on the surface of Miranda, suggested by Bauer et al. (2002) on the basis of telescopic observations, is lacking. The major moons also differ from other major satellites around giant planets in that they have very different insolation patterns, with their poles directed towards the Sun during solstice, owing to the large obliquity of the planet. Also, Oberon lies outside of the magnetosphere (depending on season), and Titania is sometimes outside the magnetosphere depending on the upstream solar wind conditions (Fig. 6), but Miranda, Ariel and Umbriel orbit within the magnetosphere and hence space weathering should have modified their surface properties, causing particles to be ejected from their surfaces.

The observations performed during the flyby of Voyager 2 revealed surprising amounts of geological activity on these moons, possibly involving cryovolcanic processes. As can be seen from

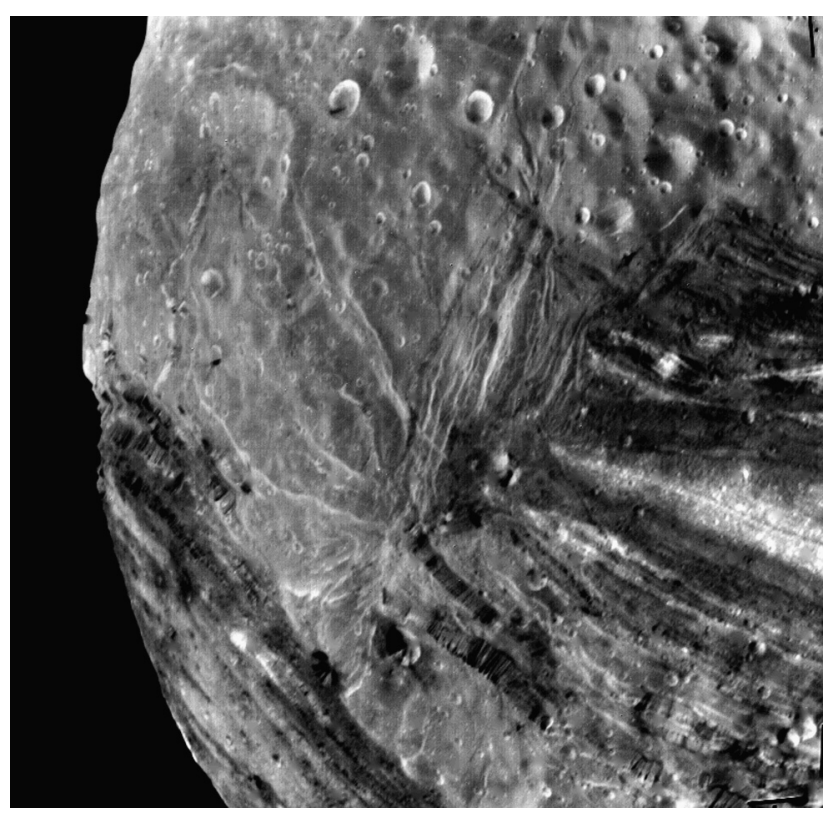

Fig. 10. Miranda's striking geological features. Credit: NASA/JPL.
Fig. 10, Miranda exhibits striking structural geology, despite its small size (472 km in diameter), with ridges and grooves that may be the result of internal differentiation processes (Janes and Melosh, 1988) or the surface expression of large-scale upwelling plumes (e.g. Pappalardo et al., 1997). Similar internal processes possibly occurred on the comparably-sized Enceladus in the saturnian system, before its intense surface activity and cryovolcanic plumes developed. Observations of Miranda thus provide a unique opportunity to understand how small moons can become so active (Castillo-Rogez and Lunine, 2012). Moreover, the convex floors of Ariel's graben may provide the only evidence for widespread cryovolcanism in the form of viscous extrusive cryolava flows (Croft and Soderblom, 1991; Schenk, 1991), a process that has been elusive in the Solar System, with only a few small examples documented elsewhere to date, for example, Sippar Sulcus on Ganymede (Schenk and Moore, 1995) and Sotra Patera on Titan (Lopes et al., 2013). However, only very limited observations were possible during Voyager 2's brief encounter, at which time only the southern hemispheres of the satellites were illuminated. The diversity of the medium-sized icy satellites at Uranus demonstrates the complex and varied histories of this class of object.

Very little is known about the composition of the irregular moons of Uranus yet they may hold keys for understanding the evolution of the uranian system, particularly in relation to the great collision hypothesis (e.g., Parisi et al., 2008). Photometrically, Sycorax and Caliban are redder than Uranus and its regular satellites, perhaps similar to Kuiper belt objects, Centaurs and comet nuclei (e.g., Maris et al., 2001) suggesting an origin as captured objects. Although, spectrally in the near-IR they are more difficult to interpret, and rotational effects may need to be included where the surfaces are spectrally inhomogeneous (Romon et al., 2001).

By using an imaging spectrometer in the near infrared range from $0.8 \mu \mathrm{m}$ to at least $5 \mu \mathrm{m}$, it will be possible to unveil the surface composition of the moons by identifying and mapping various chemical species (with particular emphasis on non-waterice materials, including volatiles and organics). This will ultimately enable an unprecedented correlation of surface composition with geologic units at various spatial scales. Spatially resolved chemical mapping will also help separate the relative contributions of endogenic subsurface chemistry and exogenic magnetospheredriven radiolysis across the moons (e.g., Cartwright et al., 2013), the transport of dust in the uranian system (e.g., Tosi et al., 2010) and assess the role of processes that exchanged material between the surface and subsurface.

\subsubsection{What is the origin of Uranus' moons and how have they evolved?}

As in the jovian and saturnian systems, tidal and magnetospheric interactions are likely to have played key roles in the evolution of the uranian satellite system. For instance, intense tidal heating during sporadic passages through resonances is expected to have induced internal melting in some of the icy moons (Tittemore and Wisdom, 1990; Tittemore, 1990). One such tidally induced melting event may have triggered the geological activity that led to the late resurfacing of Ariel. The two largest moons, Titania and Oberon, with diameters exceeding $1500 \mathrm{~km}$, might still harbour liquid water oceans between their outer ice shells and inner rocky cores, remnants of past melting events (Hussmann et al., 2006).

The surfaces of the five major satellites of Uranus exhibit extreme geologic diversity; however, understanding of their geologic evolution and tectonic processes has suffered greatly from incomplete Voyager image coverage (imaging restricted to the 
southern hemispheres) and only medium to low image resolutions (order of several kilometres per pixel, except for part of Miranda) which only allow characterisation of the largest geologic units in the areas that could be imaged (e.g., Croft and Soderblom, 1991). The crater size-frequency distributions of the five satellites, used as a tool for dating surface features and for constraining impactor origin, are known only for the southern hemispheres and crater sizes larger than a few kilometres (e.g. Plescia, 1987). There are also still large uncertainties in the bulk composition of the moons (e.g. Hussmann et al., 2006), which provide fundamental constraints on their origins.

High-resolution images of the satellite surfaces, which will provide key information on the ages and compositions of the surfaces and will constrain the dynamical and geologic histories that led to the observed diversity. For example, Miranda and Ariel exhibit evidence of significant endogenic geological activity. Highresolution surface mapping will enable us to determine the degree to which tectonic and cryovolcanic activity has occurred, permitting characterisation of the role played by tidal dissipation and understanding whether uranian moons have experienced internal activity similar to that at Enceladus. Mapping of the moons will help constrain the nature and timescale of this activity, and characterising the environment in their vicinity may reveal outgassing if, as at Enceladus, activity is continuing. Collisional activity amongst the irregular satellites can produce contamination of the regular satellite surfaces with material from the irregular satellites via dust transport (Schubert et al., 2010). High-resolution imagery and spectral data could reveal evidence of such processes.

Accurate astrometric measurements can also be used to quantify the influence of tidal interactions in the system at present, providing fundamental constraints on the dissipation factor of Uranus (Lainey, 2008). Gravimetric and magnetic measurements, combined with global shape data, will greatly improve the models of the satellites' interiors, bringing fundamental constraints on their bulk composition (density) and evolution (mean moment of inertia). Understanding the composition (particularly the ice-to-rock ratio) and the internal structure of the natural satellites will also enable us to understand if Uranus' natural satellite system was the original population of bodies that formed around the planet, or if they were subsequently disrupted, potentially via a giant impact that might have produced Uranus' large obliquity (Coradini et al., 2010).

Crater statistics will be crucial in determining the satellites' geological histories as well as providing critical information about the projectile flux in the outer Solar System. Near- and midinfrared spectroscopy will enable us to understand the surface composition of the moons yielding further information on their origin and evolution. Occultations will enable us to probe any tenuous atmospheres that may be present and UV spectroscopy may then lead constraints on their chemistry, with implications for the subsurface. The dayside magnetopause lies at a distance of 18 $\mathrm{R}_{\mathrm{U}}$ and, therefore, the major moons (except Oberon, and sometimes Titania) are embedded within the magnetosphere. This implies that their water-ice surfaces are eroded by magnetospheric charged particles in addition to photons and micrometeoroids. Measuring the properties of the charged particles that these moons can encounter, and the energetic neutral particles released after the ions impact the surface, will constrain the role of plasma bombardment on surface evolution. These data will constitute strong constraints to allow us to understand how satellite systems form and evolve around Ice Giants. The composition of the uranian moons will represent an essential data point in understanding the nature and origins of organic and volatile material in the outer Solar System.

Recent models of icy satellite interiors suggest the larger uranian satellites, Titania and Oberon, may contain subsurface oceans (Hussmann et al., 2006) and Miranda may be subject to recent or even ongoing activity (Castillo-Rogez and Turtle, 2012). The magnetic field induced in Europa's subsurface ocean was readily detectable by Galileo (e.g., Khurana et al., 1998) and any such signatures at Uranus are expected to be strong due to Uranus' asymmetrical field.

Remote observations of Uranus' irregular satellites can be used to search for potential genetic relationships with the irregular satellites found in other giant planet systems and thus understand the evolution of Solar System minor bodies and giant planet natural satellites. Amongst the irregular satellites, numerical simulations and photometry suggest at least two dynamical groupings: the Caliban group (Caliban, Stephano and Francisco), and the Sycorax group (Sycorax, Prospero, Setebos) with heterogeneous photometry supporting origins from particular parent bodies, and Tinculu, Margaret and Ferdinand as single objects with a different origin (Vilas et al., 2006; Grav et al., 2004; Sheppard et al., 2005). However, the photometric and spectroscopic observations are not consistent and new observations are required to understand the origins of these objects and their relationship to Uranus' great collision (Maris et al., 2007; Parisi et al., 2008).

\subsection{Uranus' aeronomy, aurorae, and highly asymmetrical magnetosphere}

The configuration of all the planetary magnetospheres in the Solar System is determined by the relative orientations of the planet's spin axis, its magnetic dipole axis, and the solar wind flow. In the general case, the angle between the magnetic dipole axis and the solar wind flow is a time-dependent quantity and varies on both diurnal and seasonal timescales. Uranus presents a particularly interesting and poorly understood case because this angle not only varies seasonally but because of Uranus' large obliquity the extent of diurnal oscillation varies with season. At solstice this angle does not vary with time and Uranus' magnetic dipole simply rotates around the solar wind flow. This is a magnetospheric configuration not found anywhere else in the Solar System. Fig. 11 illustrates the configuration of Uranus' magnetosphere near solstice, as sampled by Voyager 2 .

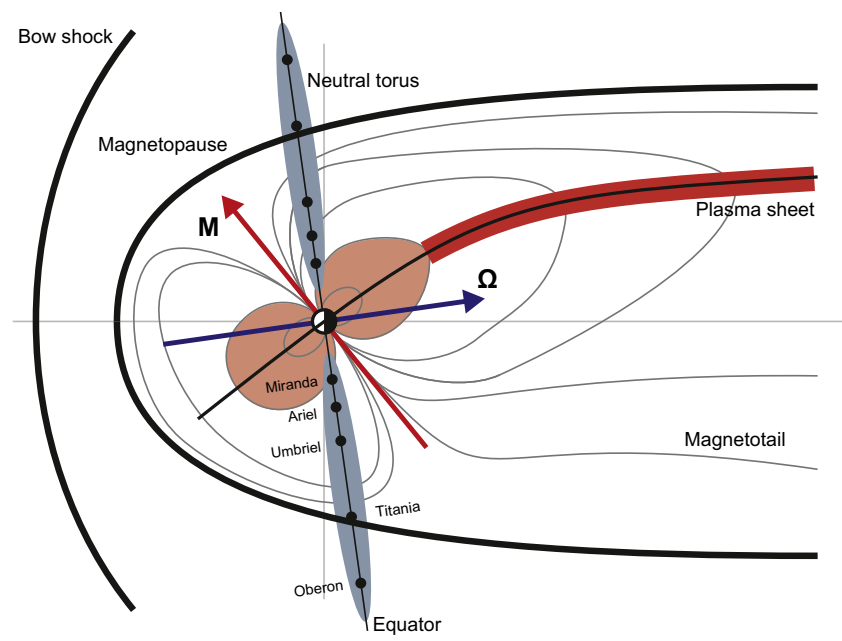

Fig. 11. Illustration of Uranus' magnetosphere at solstice as sampled by Voyager 2. The red vector indicates the magnetic dipole axis and the blue arrow the rotation axis of Uranus. Field lines are in grey and magnetopause and bow shock boundaries in heavy black lines. The plasma sheet and inner magnetospheric plasma populations are indicated by the orange and red regions respectively. The positions of natural satellites are indicated in Uranus' equatorial plane and a possible neutral torus in blue. (For interpretation of the references to colour in this figure legend the reader is referred to the web version of this article.) 
Because of this unique extreme orientation, its magnetosphere is expected to vary from a pole-on to orthogonal configuration during a uranian year and to change from an "open" (connected to the solar wind) to a "closed" configuration during a uranian day. Such a rapidly reconfiguring magnetosphere with a highly asymmetric internal magnetic field (Section 2.1.3) at its core provides a challenge for our theories of how magnetospheres work and will bring new insight in fundamental and universal magnetospheric processes. Uranus also presents a special case because of its distant location in the heliosphere where the properties of the solar wind are very different to the near-Earth environment (e.g., solar wind structures merge by propagating outwards, giving rise to successive long perturbations typically lasting $1-2$ weeks). This provides opportunities to investigate fundamental processes such as magnetic reconnection under a different parameter regime. Along with the planetary magnetic field, the ionosphere of Uranus is the internal core of the magnetosphere. Recent analysis of emissions from Uranus spanning almost 20 years (Melin et al., 2011, 2013), have revealed a phenomenon that is not seen at the other Gas Giants in our Solar System: the temperature of the ionosphere is at least partly controlled by season, such that at solstice, the upper atmosphere is more than $200 \mathrm{~K}$ hotter than at equinox, but where other influences, e.g. from the geometry of Uranus' interaction with the solar wind, are also involved.

Auroral emissions are also generated at kilometric (radio) wavelengths (1-1000 kHz), which cannot be observed from Earth or distant observers. As at other planets, UKR is thought to be generated by the Cyclotron Maser Instability. However, UKR appears to be more complex than similar radio emissions at Earth, Saturn or Jupiter and only comparable to Neptune's ones. Understanding the circumstances under which these peculiar radio emissions are generated is of prime importance for the groundbased radio detection of exoplanets with a magnetic field (essential to the development of life), particularly those with highly inclined magnetic axes with respect to the stellar flow.

Because planetary magnetospheres partially shield planets from solar energetic particles and galactic cosmic rays they have a role to play in the development of life. In order to further our understanding of how life and the platforms for life exist in the wide variety of magnetic environments in the Universe, it is vital that we make comprehensive measurements in the widest possible variety of environments. These aspects make a study of Uranus' magnetosphere a very important objective for understanding how the Solar System works and for achieving ESA's Cosmic Vision goals and those set out in the Planetary Decadal Survey. These are not only relevant for the important question of understanding how asymmetric Ice Giant magnetospheres work, but are also highly relevant in providing "ground-truth" for understanding exoplanetary magnetospheres.

\subsubsection{What is the overall configuration of the uranian magnetosphere?}

Our understanding of the uranian magnetosphere is currently essentially limited to data from the Voyager 2 flyby which provided a single snapshot where the angle of attack between the solar wind axis and the magnetic dipole axis varied between $68^{\circ}$ and $52^{\circ}$, to some extent similar to the Earth's magnetosphere. However, the near alignment of the rotation axis with the planet-Sun line during solstice means that plasma motions produced by the rotation of the planet and by the solar wind were effectively decoupled (Selesnick and Richardson, 1986; Vasyliuñas, 1986). Therefore, in contrast with Jupiter and Saturn, solar wind plasma may be able to penetrate deep within the magnetosphere despite the planet being a fast oblique rotator, although there is evidence for some shielding in the inner magnetosphere (McNutt et al., 1987; Selesnick and McNutt, 1987; Sittler et al., 1987). This may result in short residence times for magnetospheric plasma produced deep within the magnetosphere and may limit the amount of plasma trapping inside the magnetosphere and consequently the amount of charged particle acceleration (e.g., Cheng, 1987). Proton and electron radiation belts (with energies up to tens of $\mathrm{MeV}$ ) albeit slightly less intense than those at Saturn were also observed in the inner magnetosphere of Uranus (Cheng, 1991) but their diurnal and seasonal variability is largely unknown.

The significant asymmetries in the magnetosphere result in largescale diurnal reconfigurations of the system on timescales of hours, resulted in a twisted magnetotail topology (Behannon et al., 1987; Tóth et al., 2004; Arridge, in press). The main plasma sources, transport modes and loss processes in the uranian magnetosphere, and the modes of interaction (pick-up, sputtering, and charge exchange) between the magnetospheric plasma and the rings and moons of Uranus are also largely unknown. The configuration and dynamics of the uranian magnetosphere at equinox are entirely unknown and it is not clear if this will result in a fairly quiescent magnetosphere such as Neptune, or a more rotationally dominated magnetosphere like Jupiter or Saturn. Recent observations and theoretical work suggest a limited role for solar wind-driven dynamics at equinox (Lamy et al., 2012; Cowley, 2013) and solar wind-magnetosphere coupling via magnetic reconnection that varies strongly with season and solar cycle (Masters, 2014).

\subsubsection{What are the characteristics and origins of the uranian aurorae?}

Aurorae are the most striking diagnosis of the magnetosphere dynamics, as they can be traced back to the currents generated by the magnetospheric interactions. Several kinds of interactions have been characterised at Earth, Jupiter and Saturn, but the Uranus optical and radio aurorae, as they are known from Voyager 2 observations seem to indicate new kinds of interactions. The charged particles responsible for both optical and radio auroral

a

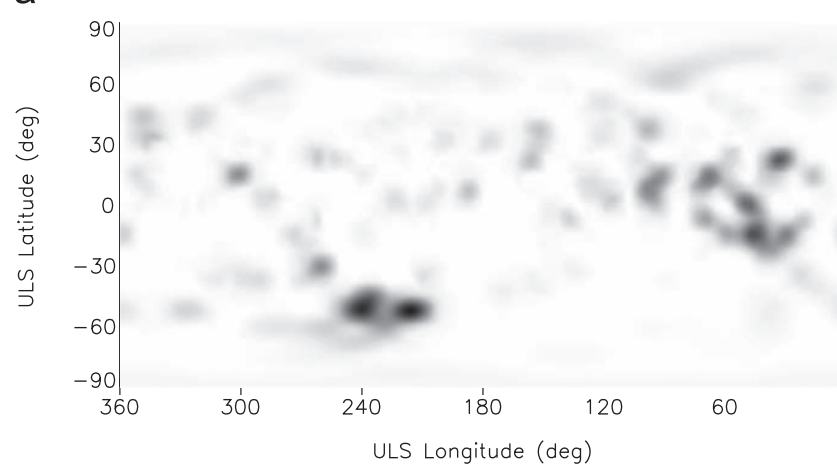

b

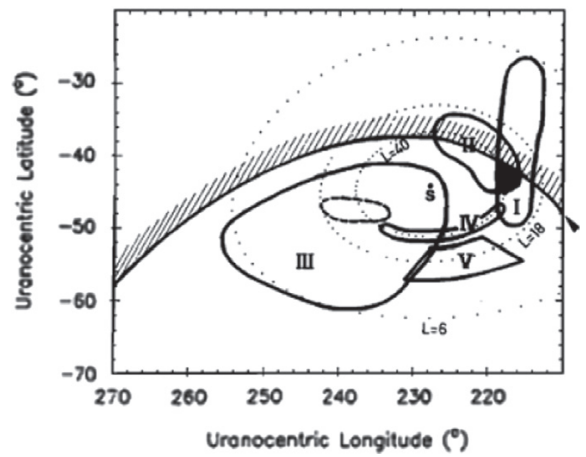

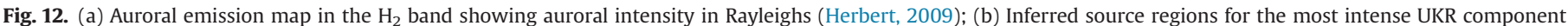
(Zarka and Lecacheux, 1987). From Arridge et al. (2012). 
emissions and their source regions are also unknown. A study of the uranian auroral regions can also lead to information on the thermosphere due to atmospheric sputtering produced by auroral particle precipitation. Such sputtered particles can be monitored by a neutral particle detector.

There has only been one spatially resolved observation of the UV aurora of Uranus (Herbert, 2009), using a mosaic of Voyager 2 UV observations mapping emission from $H$ Lyman- $\alpha$ and EUV $\mathrm{H}_{2}$ band emission (Fig. 12a). The emission appeared patchy and was generally centred on the magnetic poles, with the emission being the brightest about midnight magnetic local time. There have been subsequent attempts to observe the aurora in both the far ultraviolet using the Hubble Space Telescope (HST) (Ballester, 1998) and in the IR using ground-based telescopes (e.g., Trafton et al., 1999). Uranus' aurorae was recently redetected in the UV using HST (Lamy et al., 2012) and revealed a radically different set of auroral processes controlled by the interaction between the magnetosphere and the solar wind (Cowley, 2013), and raising important questions on the generation of planetary auroral emissions and possible secular drift of Uranus' intrinsic magnetic field.

The UKR components, which indicate different active regions in the magnetosphere, divide into two categories: (i) "bursty" ( $<10 \mathrm{~min}$ ) emissions comparable to that at Earth and Gas Giants, and (ii) "smooth emissions" which are time-stationary emissions (lasting for hours) specific to Ice Giants (Zarka and Lecacheux, 1987). These latter components require a continuous source of free energy that has not yet been identified and is apparently maintained in a highly variable magnetosphere (Fig. 12b). New radio observations with a modern instrumentation will provide wave properties that were inaccessible to Voyager 2, such as the wave direction and polarisation. Continuous remote observations of UKR and in situ measurements within their various source regions will provide essential information to understand the origin and characteristics of the variety of known uranian radio components and search for new components.

Recent calculations show that new ground-based radio telescopes could detect radio emissions from hot Jupiters (Zarka, 2007). Unlike our Solar System, eccentric and complex orbital characteristics appear to be common in other planetary systems, so that the understanding of radio emission produced by Uranus could have profound importance in interpreting future radio detections of exoplanets.

\subsubsection{How does solar wind-magnetosphere-ionosphere coupling work at ice giants?}

The uranian magnetosphere interacts with a fast magnetosonic Mach number and high-beta solar wind, which is an important plasma regime in which to understand magnetic reconnection (e.g., Masters, 2014), however, Richardson et al. (1988) have reported Voyager 2 observations suggesting the presence of periodic reconnection near the magnetopause. Evidence of dynamics, similar to Earth-like substorm activity but possibly internallydriven, was also reported at Uranus by Mauk et al. (1987) and Sittler et al. (1987) which indicate that important energy sources need to be quantified, including the energy input from the solar wind. We do not know how the solar wind-magnetosphere interaction is interrupted and modulated by the diurnally changing geometry. Together, Uranus' ionosphere and internal magnetic field act as the inner boundary condition for the magnetosphere. Models indicate that Uranus' ionosphere is dominated by $\mathrm{H}^{+}$at higher altitudes and $\mathrm{H}_{3}^{+}$lower down (Capone et al., 1977; Chandler and Waite, 1986; Majeed et al., 2004), produced by either energetic particle precipitation or solar ultraviolet (UV) radiation. It seems likely that a key component of the required additional heating is driven by particle precipitation and/or the way in which varying magnetospheric configurations couple with the upper atmosphere.
Understanding how the aurorae of Uranus respond to changes in the solar wind is essential to understanding the Solar Wind interaction with giant planets more generally. While these responses are well studied for the Earth, the situation for the outer planets is less well understood, partly due to the lack of dedicated deep space solar wind monitors. Recent theoretical work (Cowley, 2013) has argued for distinct differences in magnetotail processes between equinox and solstice, thus providing a framework for the interpretation of new auroral images and demonstrating the need for new in situ measurements. The magnetosphere of Uranus was observed to be the site of intense plasma-wave activity with remarkably intense whistler mode emissions (Kurth et al., 1991). The role of wave-particle interactions for the magnetosphereionosphere coupling and the generation of Uranus' auroral emissions, as well as for the overall energy budget of the magnetosphere require further consideration.

\subsection{Cruise phase science in the outer heliosphere}

A mission to Uranus naturally involves a relatively long duration interplanetary transfer $(\sim 15$ years, see Section 3.1$)$. However, this presents an opportunity to undertake studies of the outer heliosphere, minor Solar System bodies, and fundamental physics of the gravitational interaction.

\subsubsection{Physics of the interplanetary medium}

The structure of the heliosphere originates in the structure of the solar magnetic field and is strongly modified by the solar corona. There are a range of important questions on how this structure is further modified and processed in the heliosphere and goes on to modulate the cosmic ray flux in the inner heliosphere, on the generation of turbulence, and how minor bodies interact with the heliosphere. One of the major issues of the physics of interplanetary medium is to understand the mechanisms of energy dissipation. Injected with large spatial scales by the Sun, the energy is transferred to smaller scales (ion/electron), where it is dissipated as heat. Measurements made by the Voyager probes have revealed variations of the exponents of the power law of certain parameters (e.g., speed, magnetic field, density) with distance from the Sun, suggesting regime change in the process of energy transfer (Burlaga et al., 1997). Few observations of the heliospheric environment beyond $10 \mathrm{AU}$ have been made since Pioneer 10 and 11, Voyagers 1 and 2, and New Horizons with very few observations made at solar maximum. Energetic particle observations during cruise out to 19.2 AU will facilitate further study of the interaction between the outer heliosphere and interstellar medium, as carried out by Cassini at $9.5 \mathrm{AU}$ and Interstellar Boundaries Explorer (IBEX) at $1 \mathrm{AU}$. A cruise phase to Uranus also allows the characterisation of interplanetary and interstellar dust with radial distance from the Sun.

Interstellar dust penetrates deep into the heliosphere and does provide the unique opportunity for an in situ analysis of its dynamical and compositional information which varies with distance from the Sun and with the solar cycle. The current data set including composition information of interplanetary and interstellar grains is very limited. Only Cassini carried a spectrometer and the pointing profile during the cruise phase was not optimised for interplanetary and interstellar dust measurements. A mission to Uranus would help to close this knowledge gap which is essential to understand Solar System formation and evolution.

\subsubsection{Fundamental physics and departures from General Relativity}

General Relativity has been confirmed by all the precision experiements performed so far. But experimental tests leave open 
windows for deviations from this theory at short (Antoniadis et al., 2011) or long (Reynaud and Jaekel, 2005) distances. General Relativity is also challenged by observations at galactic and cosmic scales. The rotation curves of galaxies and the relation between redshifts and luminosities of supernovae deviate from the predictions of the theory. These anomalies are interpreted as revealing the presence of so-called "dark matter" and "dark energy". Their nature remains unknown and, despite their prevalence in the energy content, they have not been detected up to now by other means than gravitational measurements.

Given the immense challenge posed by these large scale observations, in a context dominated by the quest for the nature of dark matter and dark energy, it is important to explore every possible explanation including the hypothesis that General Relativity could not be the correct description of gravitational phenomena at large scales (Aguirre et al., 2001; Nojiri and Odintsov, 2007). Extending the range to which gravity is probed is therefore essential to bridge the gap between experiments in the Solar System and astrophysical or cosmological observations (Turyshev, 2008). In this respect, as has been customary for the deep-space missions, the spacecraft is seen as a test mass (almost) freely falling in the Solar System gravitational environment. High precision microwave tracking data (as that offered by $\mathrm{K}_{\mathrm{a}}$-band) besides the standard navigation operations - can be analysed searching for possible deviations from the trajectory predicted by General Relativity. Of primary importance in exploiting the information content of these data will be a proper modelling of spacecraft dynamics. Combining radio-science and acceleration measurements not only improves the precision and quality of spacecraft navigation but also allows us to remove, as fully as possible, the systematic effects of non-gravitational forces acting on the spacecraft (Iafolla et al., 2010). These scientific goals are intimately connected to the planetary science goals since gravitation is directly connected to planetary ephemeris (Fienga et al., 2010) as well as to the origins of the Solar System (Blanc et al., 2005).

\subsubsection{Small icy bodies in the outer heliosphere}

Centaurs and trans-Neptunian objects (TNOs) are the most pristine and less-processed remnants of the icy debris that formed the outer planets and are the most observable Solar System analogues for debris disks observed around other stars. Centaurs and TNOs are widely thought to be objects scattered from the Kuiper belt that may evolve into short-period comets (Cruikshank, 2005). Surveys of surface properties indicate potential genetic links between TNOs, Centaurs, comets and water-rich asteroids. Some of these objects show evidence of episodic cometary-like behaviour, for example 2060 Chiron (Luu et al., 2000). No mission is currently planned to visit a Centaur/TNO but the cruise phase for a mission to Uranus provides an opportunity to visit such an object en route to Uranus. As a proof-of-concept we took a nominal launch date of 2028 and searched for Centaurs/TNOs that might be accessible for a flyby en route to Uranus and found that objects 2060 Chiron, 2010 KG43, 330759 and 2007 TB434 could potentially be visited en route. Comets and asteroids are also natural targets for flybys during the cruise phase. Naturally further mission study is required to investigate this in more detail.

\section{Strawman mission concept}

In terms of mission options, the primary trade space is between an orbiter and a flyby mission. Some goals can be partially satisfied with a flyby mission but to fully answer the questions laid out in Section 2 requires an orbiting platform to make repeated observations of Uranus and its planetary system. There exists an additional trade space between enhanced remote sensing instrumentation and an entry probe. But some science questions (Sections 2.1.1, 2.1.5 and 2.1.6) can only be answered with an atmospheric entry probe to a $>5$ bar depth. For the purposes of the Uranus white paper, the outline mission concept consisted of an orbiter in a polar science orbit with an atmospheric entry probe. A specific prime science phase duration was not determined and depends sensitively on a number of factors, including the instrument payload and science orbits. However, we note that Arridge et al. (2012) and Hubbard (2010) considered 620- and 431-day science phase durations, respectively. In some cases the exploration of Uranus can be seen as easier than Saturn, for example, particularly for the planet itself since a spacecraft can easily inject into a polar orbit. This potentially makes the study of moons more difficult than Cassini-Huygens at Saturn. Novel solutions to return science data will be required due to the lower communications rates from $19 \mathrm{AU}$ compared to Cassini. Table 2 illustrates the strawman instrument suite, composed of high technology readiness level (TRL) instruments.

\subsection{Interplanetary transfers and orbital entry}

Interplanetary transfers to Uranus have been studied in a number of mission analyses (Arridge et al., 2012; Hubbard, 2010) and demonstrate the feasibility of a mission to Uranus with current technology and including an interplanetary transfer between 10 and 16 years. The mission is feasible with high TRL conventional chemical propulsion and solar-electric propulsion employing ion engines provides potential gains in margins, available $\Delta v$ and platform/instrumentation mass (e.g., Hubbard, 2010). Concepts involving lower TRL technology, such as E-sails (e.g., Janhunen, 2004; Janhunen et al., this issue), would be naturally beneficial.

The range of acceptable periapsis latitudes and radial distances at Uranus orbit insertion are limited due to the largely unknown ring plane hazards. This can be mitigated with a high latitude periapsis and orbit insertion manoeuver followed by a ring plane

Table 2

Strawman scientific payload for a Uranus orbiter/entry probe mission.

\begin{tabular}{ll}
\hline Instrument & Heritage \\
\hline Orbiter & \\
Magnetometer & Cassini/MAG \\
Plasma and particle package & Solar Orbiter \\
& Rosetta/RPC-IES \\
Radio and plasma wave experiment & New Horizons/PEPPS \\
& Cassini/RPWS \\
Microwave radiometer & Bepi-Colombo/MMO/PWI \\
Thermal infrared bolometer & Juno/MWR \\
& LRO/Diviner \\
Visual and near-infrared mapping spectrometer & Bepi-Colombo (detectors) \\
& New Horizons/RALPH \\
& Rosetta/VIRTIS \\
Ultraviolet imaging spectrometer & Dawn/VIR \\
& Bepi-Colombo/PHEBUS \\
Visible camera & Mars Express/SPICAM-UV \\
& Mars Express/HRSC \\
Radio science experiment & New Horizons/LORRI \\
Accelerometer & Venus Express/VeRa \\
Dust detector & Rosetta/RSI \\
Probe & CHAMP/STAR \\
Mass spectrometer & Cassini/CDA \\
Nephelometer & \\
Radio science & Huygens/GCMS \\
Accelerometer & Galileo/GPMS \\
\hline & Galileo/NEP \\
& Huygens/DWE \\
& Huygens/HASI \\
\hline & \\
& \\
& \\
& \\
& \\
&
\end{tabular}


crossing beyond $52,000 \mathrm{~km}$, inside of which are the main ring plane hazards. Although aerocapture is a natural technology to use at orbit insertion, the atmosphere of Uranus is poorly understood and aerocapture is low TRL technology, thus representing a highrisk option.

Uranus' large obliquity permits a range of insertion orbital inclinations, from equatorial to polar. The lack of large natural satellites does not permit low-fuel inclination changes and so an initial polar orbit is preferred since these are ideal for studies of Uranus' interior, atmosphere and magnetic field that are required to meet the goals in section two.

\subsection{Atmospheric entry probe}

An atmospheric entry probe for Uranus has been studied by the ESA Concurrent Design Facility (Biesbroek et al., 2010), which led to a $312 \mathrm{~kg}$ entry probe (including 20\% system margin) using a dedicated carrier platform. The mission concept we outline would involve using the Uranus orbiter as a carrier and communications relay. The instrumentation for such an entry probe is all available within Europe and is high TRL. The key technology development requirement is the thermal protection system for the entry probe. However, such a probe might be provided via international cooperation and has been studied by NASA (Agrawal et al., 2014).

\subsection{Critical issues}

Voyager 2 found that the radiation belts of Uranus were similar to Earth and Saturn in terms of intensity and so the radiation environment of Uranus is not judged to be a significant mission driver. Arridge et al. (2012) estimated the radiation dose for the Uranus Pathfinder mission concept using the SHEILDDOSE-2 software and found that the largest dose came from the cruise phase (18 krad behind $4 \mathrm{~mm}$ of $\mathrm{Al}$ ) with only $2 \mathrm{krad}$ per science orbit based on a scaled model of Earth's magnetosphere. The main critical issues for a Uranus mission are electrical power (Section 3.3.1), thermal control (Section 3.3.2), telemetry (Section 3.3.3), and cruise phase duration (Section 3.3.4).

\subsubsection{Electrical power}

The key technology development requirement for a mission to Uranus is the provision of sufficient electrical power at 19.2 AU. Scaling ESA's Rosetta mission solar arrays out to Uranus we estimate that providing $400 \mathrm{~W}_{\mathrm{e}}$ at Uranus would require $800 \mathrm{~m}^{2}$ solar arrays producing system level issues associated with a large launch mass and spacecraft moment of inertia. At present a nuclear (radioisotope) power source (RPS) is the only viable alternative. ${ }^{241} \mathrm{Am}$ is the isotope that has been selected for ESA RPS devices that are currently in the developmental stage (see O'Brien et al. (2008), Arridge et al. (2012), and Sarsfield et al. (2013) for a discussion of issues relating to the use of ${ }^{241} \mathrm{Am}$ ). To provide target electrical power of $400 \mathrm{~W}_{\mathrm{e}}$ at Uranus after 14 years flight time would require a total RPS system mass of $200 \mathrm{~kg}$ (excluding any maturity margin) based on a radioisotope thermoelectric generator (RTG) design with a specific power of $2.0 \mathrm{~W}_{\mathrm{e}} / \mathrm{kg}$, compared with $2.9 \mathrm{~W} / \mathrm{kg}$ (at the beginning of mission) for a NASA multi-mission RTG using ${ }^{238} \mathrm{Pu}$ (Abelson et al., 2005). Although the development of such technology presents a schedule and cost risk, this is currently under development as part of an ESA development programme and with sustained investment should reach a higher TRL in the 2025-2035 timeframe. In addition to RTG systems Stirling generator-based nuclear power sources are also under development in Europe and specific power values will be determined at the end of an active ESA study. Stirling-based solutions could offer an alternative option with a higher specific power on similar timescales to the RTG programme.

\subsubsection{Thermal control}

Thermal control is an important driver for every mission. Extreme differences in thermal environment between the inner heliosphere (for trajectories involving Venus gravity assists) and Uranus, and due to the continuous supply of thermal energy from RPS units present the most important issues. Such thermal control issues can be adequately managed by modifying existing designs from Rosetta and Mars/Venus Express. Thermal control for a Uranus mission was studied using ThermXL, based on a spacecraft of a similar size to Mars Express and including waste heat dissipation from the RPS. We estimated that electrical heaters consuming around $50 \mathrm{~W}$ would be sufficient to maintain an internal spacecraft temperature of $-30{ }^{\circ} \mathrm{C}$ against losses to space. Waste electrical power from the RPS can be dissipated via externally- or internally-mounted shunt resistors but could impact on overall system design. Radioisotope heater units based on ${ }^{241} \mathrm{Am}$ can offer distributed heating solutions, with each unit generating between $1 \mathrm{~W}$ and $5 \mathrm{~W}$ of thermal power. This would reduce the requirement to distribute waste heat from RTGs of Stirling-based systems and reduce demands for electrical heating. The use of these heaters or waste heat from RPS solutions should form part of a future trade-off study.

\subsubsection{Telemetry rates}

To answer the questions in Section 1 requires significant volumes of data to be returned over $\lesssim 20.9$ AU. Downlink transmissions over $\mathrm{K}_{\mathrm{a}}$-band to ESA's Cebreros station, using a $4 \mathrm{~m}(3 \mathrm{~m})$ high gain antenna, with a $100 \mathrm{~W}$ power input to the transmitter on an orbiter with a pointing accuracy of $0.05^{\circ}$ (comparable to the Cassini orbiter) will achieve a downlink rate of $4.5 \mathrm{kbit} / \mathrm{s}(1.5 \mathrm{kbit} / \mathrm{s})$ at $10^{\circ}$ elevation and $7.2 \mathrm{kbit} / \mathrm{s}(2.4 \mathrm{kbit} / \mathrm{s})$ at $80^{\circ}$ elevation, equivalent to $\sim 170$ ( $\sim 60$ ) Mbit per $8 \mathrm{~h}$ downlink. Using ground station arrays and utilising larger dishes (for example, via collaboration with NASA to use the Deep Space Network) will naturally increase these data volumes. These data volumes should be sufficient to achieve the essential science goals.

\subsubsection{Long cruise phase duration}

To reduce cruise phase costs a Uranus mission might employ hibernation modes (similar to those used on New Horizons and Rosetta) to minimise operations costs and ground station antenna usage. A cruise phase science programme, as outlined in Section 2, will periodically enable the platform and science instruments to be utilised and tested. In addition, special hibernation modes would permit some instruments to collect low-rate cruise phase science data. The use of high TRL technology and minimising the cruise phase operations will reduce demands on spacecraft platform components, reduce the mission cost-at-completion, and lessen demands on the electrical power system.

\subsection{International cooperation}

Such a large and significant interplanetary mission would naturally benefit from collaboration with other space agencies. The white paper had broad support from scientists funded by NASA and JAXA, and within Europe. Uranus has been named a priority by NASA as recommended by the Planetary Decadal Survey. In the context of international cooperation, a partner agency may provide an atmospheric entry probe, provide instruments for the orbiter/entry probe thus lessening the demand on ESA member states, or may provide a launch vehicle. 


\section{Acknowledgements}

CSA and LNF were supported by Royal Society University Research Fellowships. C.S.A. thanks O. Bedworth, B. Jacobson, and J.-P. Lebreton for useful discussions and comments on the manuscript.

\section{References}

Abelson, R.D., Balint, T.S., Coste, K., Elliott, J.O., Randolph, J.E., Schmidt, G.R., Schriener, T. Shirley, J.H., Spilker, T.R., 2005. Expanding Frontiers with Standard Radioisotope Power Systems. Jet Propulsion Laboratory, JPL-28902.

Agrawal, P., Allen, G.A., Hwang, H.H., Marley, M.S., McGuire, M.K, Garcia, J.A., Sklyanskiy, E., Huynh, L.C., Moses, R.W., 2014. Atmospheric entry studies for Uranus. 11th International Planetary Probe Workshop, June 16-20, 2014 Pasadena, CA. LPI Contribution number 1795, ID 8028.

Aguirre, A., Burgess, C.P., Friedland, A., Nolte, D., 2001. Astrophysical constraints on modifying gravity at large distances. Class. Quant. Grav. 18 (23), R223-R232. http://dx.doi.org/10.1088/0264-9381/18/23/202.

Antoniadis, I., Baessler, S., Büchner, M., Fedorov, V.V., Hoedl, S, Lambrecht, A., Nesvizhevsky, V.V., Pignol, G., Protasov, K.V., Reynaud, S., Sobolev, Yu., 2011 Short-range fundamental forces. C.R. Phys. 12 (8), 755-778. http://dx.doi.org 10.1016/j.crhy.2011.05.004.

Arridge, C.S., Agnor, C.B., André, N., Baines, K.H., Fletcher, L.N., Gautier, D. Hofstadter, M.D., Jones, G.H., Lamy, L., Langevin, Y., Moussis, O., Nettelmann, N., Russell, C.T., Stallard, T., Tiscareno, M.S., Tobie, G., Bacon, A., Chaloner, C., Guest, M., Kemble, S., Peacocke, L., Achilleos, N., Andert, T., Banfield, D., Barabash, S., Barthelemy, M., Bertucci, C., Brandt, P., Cecconi, B., Chakrabarti, S. Cheng, A., Christensen, U., Christou, A., Coates, A., Collinson, G., Cooper, J.F., Courtin, R., Dougherty, M.K., Ebert, R.W., Entradas, M., Fazakerley, A.N., Fortney, J.J., Galand, M., Gustin, J., Hedman, M., Helled, R., Henri, P., Hess, S. Holme, R., Karatekin, O., Krupp, N., Leisner, J., Martin-Torres, J., Masters, A., Melin, H., Miller, S., Müller-Wodarg, I., Noyelles, B., Paranicas, C., de Pater, I., Pätzold, M., Prangé, R., Quémerais, E., Roussos, E., Rymer, A.M., Sénchez-Lavega, A., Saur, J., Sayanagi, K.M., Schenk, P., Schubert, G., Sergis, N., Sohl, F., Sittler Jr., E. C., Teanby, N.A., Tellmann, S., Turtle, E., Vinatier, S., Wahlund, J.-E., Zarka, P., 2012. Uranus Pathfinder: exploring the Origins and Evolution of Ice Giant Planets. Exp. Astron., 33; pp. 753-791. http://dx.doi.org/10.1007/s10686-011-9251-4.

Arridge, C.S. The magnetotails of Uranus and Neptune. In: AGU Chapman Monograph on Magnetotails throughout the Solar System (in press).

Atreya, S.K., Sandel, B.R., Romani, P.N., 1991. Photochemistry and vertical mixing. In: Matthews, M.S., Bergstralh, J.T., Miner, E.D. (Eds.), Uranus. University of Arizona Press, Tucson, Arizona, USA.

Bagenal, F., 1992. Giant planet magnetospheres. Annu. Rev. Earth Planet. Sci. 20, 289-328.

Baker, D., et al., 2013. Solar and Space Physics: A Science for a Technological Society. Committee for a Decadal Strategy in Solar and Space Physics: National Research Council. The National Academies Press, Washington, DC, ISBN: 978-0-309-16428-3.

Ballester, G.E., 1998. Magnetospheric interactions in the major planets. In: Wamsteker, W., Gonzalez Riestra, R. (Eds.), Ultraviolet Astrophysics Beyond the IUE Final Archive, Proceedings of the Conference held in Sevilla, Spain from 11 to 14 November 1997, ESA SP, vol. 413. ESA Publications Division, p. 21.

Bauer, J.M., Roush, T.L., Geballe, T.R., Meech, K.J., Owen, T.C., Vacca, W.D., Rayner, J.T Jim, K.T.C., 2002. The near infrared spectrum of Miranda: evidence of crystalline water ice. Icarus 158 (1), 178-190. http://dx.doi.org/10.1006/icar.2002.6876.

Behannon, K.W., Lepping, R.P., Sittler Jr., E.C., Ness, N.F., Mauk, B.H., Krimigis, S.M McNutt Jr., R.L., 1987. The magnetotail of Uranus. J. Geophys. Res. 92 (A13) 15354-15366.

Biesbroek, R., Coste, P., Allgranza, C., Patti, S., Stephenson, K., Alvarez, O., Mangunsong, S., de Wilde, D., Lamboglia, E., Monteleone, C., Schonenborg, R., Voirin, T., Drai, R., Timm, R., Falkner, P., Rebuffat, D., Gehler, M., Tomuta, D., Pickering, A 2010. Concurrent Design Facility Study Report PEP (S.U.N.) Planetary Entry Probes to Saturn, Uranus and Neptune. CDF-106(B), European Space Agency.

Blanc, M., Moura, D., Alibert, Y., André, N., Atreya, S.K., Bara, I., Barthélémy, M., Barucci, A., Beebe, R., Benz, W., Bézard, B., Bockelée-Morvan, D., Bolton, S.J., Brown, R.H., Chanteur, G., Colangeli, L., Coradini, A., Doressoundiram, A. Dougherty, M. Drossart, P., Festou, M. Flamini, E., Fulchignoni, M. Galand, M., Gautier, D., Gombosi, T., Gruen, E., Guillot, T., Kallenbach, R. Kempf, S., Krimigis, T., Krupp, N., Kurth, W., Lamy, P., Langevin, Y., Lebreton, J.-P., Leger, A., Louarn, P., Lunine, J., Matson, D., Morbidelli, A Owen, T., Prangé, R., Raulin, F., Sotin, C., Srama, R., Strobel, D.F., Thomas, N., Waite, H., Witasse, O., Zarka, P., Zarnecki, J., 2005. Tracing the origins of the Solar System. In: Favata, F., Sanz-Forcada, J., Giménez, A., Battrick, B. (Eds.) Proceedings of the 39th ESLAB Symposium on Trends in Space Science and Cosmic Vision 2020. ESA Special Publication 588, p. 213.

Boué, G., Laskar, J., 2010. A collisionless scenario for Uranus tilting. Astrophys. J. 712, L44 http://dx.doi.org/10.1088/2041-8205/712/1/L44.

Broadfoot, A.L., Herbert, F., Holberg, J.B., Hunten, D.M., Kumar, S., Sandel, B.R., Shemansky, D.E., Smith, G.R., Yelle, R.V., Strobel, D.F., Moos, H.W., Donahue, T. M., Atreya, S.K., Bertaux, J.L., Blamont, J.E., Mcconnell, J.C., Dessler, A.J., Linick, S. Springer, R., 1986. Ultraviolet spectrometer observations of Uranus. Science 233, 74-79. http://dx.doi.org/10.1126/science.233.4759.74.
Briois, C., Thissen, R., Engrand, C., Altwegg, K., Bouabdellah, A., Boukrara, A., Carrasco, N., Chapuis, C., Cottin, H., Grün, E., Grand, N., Henkel, H., Kempf, S., Lebreton, J.-P., Makarov A., Postberg, F., Srama, R., Schmidt, J., Szopa, C. Thirkell, L., Tobie, G., Wurz, P., Zolotov, M.E., 2013. Dust OrbiTrap Sensor (DOTS) for in-situ analysis of airless planetary bodies. In: Proceedings of the 44th Lunar and Planetary Science Conference, March 18-22, 2013, The Woodlands, TX. LPI Contribution 1719, p. 2888.

Brown, R.A., Cruikshank, D.P., 1983. The uranian satellites: surface compositions and opposition brightness surges. Icarus 55 (1), 83-92. http://dx.doi.org/ 10.1016/0019-1035(83)90052-0.

Burlaga, L.F., Ness, N.F., Belcher, J.W., 1997. Radial evolution of corotating merged interaction regions and flows between 14 AU and 43 AU. J. Geophys. Res. 102, 4661-4672.

Capone, L.A., Whitten, R.C., Prasad, S.S., Dubach, J., 1977. The ionospheres of Saturn, Uranus, and Neptune. Astrophys. J. 215, 977-983. http://dx.doi.org/10.1086/155434.

Cartwright, R., Emery, J.P., Rivkin, A., Trilling, D., 2013. Near-infrared spectroscopy of Uranian satellites: searching for carbon dioxide ice on Umbriel, Titania, and Oberon. In: Proceedings of the 44th Lunar and Planetary Science Conference, March 18-22, 2013, Woodlands, TX. LPI Contribution nNo. 1719, p.1195.

Castillo-Rogez, J., Turtle, E.P., 2012. Comparative planetology between the uranian and saturnian satellite systems - focus on Ariel. American Astronomical Society DPS meeting \#44, \#104.02.

Castillo-Rogez, J.C., Lunine, J.I., 2012. Tidal response of Titan's interior models consistent with Cassini-derived constraints. In: Proceedings of the 43rd Lunar and Planetary Science Conference, March 19-23, 2012, Woodlands, TX. LPI Contribution 1659, p. 1707.

Cavalié, T., Moreno, R., Lellouch, E., Hartogh, P., Venot, O., Orton, G.S., Jarchow, C., Encrenaz, T., Selsis, F., Hersant, F., Fletcher, L.N., 2014. First submillimeter observation of CO in the stratosphere of Uranus. Astron. Astrophys. 562, http: //dx.doi.org/10.1051/0004-6361/201322297 (article number A33).

Chandler, M.O., Waite, J.W., 1986. The ionosphere of Uranus-a myriad of possibilities. Geophys. Res. Lett. 13, 6-9. http://dx.doi.org/10.1029/GL013i001p00006.

Cheng, A.F. 1987. Proton and oxygen plasmas at Uranus. J. Geophys. Res. 92 (A13), 15309-15314.

Cheng, A.F., 1991. Energetic particles at Uranus. In: Matthews, M.S., Bergstralh, J.T., Miner, E.D. (Eds.), Uranus. University of Arizona Press, Tucson, Arizona, USA.

Christensen, U.R., Tilgner, A., 2004. Power requirement of the geodynamo from ohmic losses in numerical and laboratory dynamos. Nature 429 (6988), 169-171. http://dx.doi.org/10.1038/nature02508.

Colwell, J.E., Esposito, L.W., 1993. Origins of the rings of Uranus and Neptune. II Initial conditions and ring moon populations. J. Geophys. Res. 98 (E4), 7387-7401. http://dx.doi.org/10.1029/93JE00329.

Coradini, A., Magni, G., Turrini, D., 2010. From gas to satellitesimals: disk formation and evolution. Space Sci. Rev. 153, 411-429. http://dx.doi.org/10.1007/s11214009-9611-9.

Cowley, S.W.H., 2013. Response of Uranus' auroras to solar wind compressions at equinox. J. Geophys. Res. 118, 2897-2902. http://dx.doi.org/10.1002/jgra.50323.

Crida, A., Charnoz, S., 2012. Formation of regular satellites from ancient massive rings in the solar system. Science 338 (6111), 1196. http://dx.doi.org/10.1126/ science.1226477.

Croft, S.K.. Soderblom, L.A., 1991. Geology of the Uranian satellites. In: Bergstrahl, J.T. Miner, M.S., Matthews, M.S. (Eds.), Uranus. University of Arizona Press, Tucson, pp. 561-628.

Cruikshank, D.P., 2005. Triton, Pluto, Centaurs, and trans-Neptunian bodies. Space Sci. Rev. 116, 421-439. http://dx.doi.org/10.1007/s11214-005-1964-0.

Cuzzi, J.N., Burns, J.A., Charnoz, S., Clark, R.N., Colwell, J.E., Dones, L., Esposito, L.W., Filacchione, G., French, R.G., Hedman, M.M., Kempf, S., Marouf, E.A., Murray, C. D., Nicholson, P.D., Porco, C.C., Schmidt, J., Showalter, M.R., Spilker, L.J., Spitale, J. N., Srama, R., Sremčević, M., Tiscareno, M.S., Weiss, J., 2010. An evolving view of Saturn's dynamic rings. Science 327 (5972), 1470. http://dx.doi.org/10.1126/ science.1179118.

Davis, L., Smith, E.J., 1990. New models of Saturn's magnetic field using Pioneer 11 vector helium magnetometer data. J. Geophys. Res. 91 (A2), 1373-1380.

de Pater, I., Romani, P.N., Atreya, S.K., 1991. Possible microwave absorption by H2S gas in Uranus' and Neptune's atmospheres. Icarus 91, 220-233. http://dx.doi. org/10.1016/0019-1035(91)90020-T.

de Pater, I., Lissauer, J., 2010. Planetary Sciences. Cambridge University Press, Cambridge, UK.

de Pater, I., Gibbard, S.G., Hammel, H.B., 2006a. Evolution of the dusty rings of Uranus. Icarus 180 (1), 186-200. http://dx.doi.org/10.1016/j.icarus.2005.08.011.

de Pater, I., Hammel, H.B., Gibbard, S.G., Showalter, M., 2006b. New dust belts of Uranus: one ring, two ring, red ring, blue ring. Science 312 (5), 92-94.

de Pater, I., Hammel, H.B., Showalter, M.R., van Dam, M.A., 2007. The dark side of the rings of Uranus. Science 317 (5846), 1888. http://dx.doi.org/10.1126/ science 1148103.

de Pater, I., Sromovsky, L.A., Hammel, H.B., Fry, P.M., LeBeau, R.P., Rages, K., Showalter, M.R., Matthews, K., 2011. Post-equinox observations of Uranus: Berg's evolution, vertical structure, and track towards the equator. Icarus 215 (1), 332-345. http://dx.doi.org/10.1016/j.icarus.2011.06.022.

de Pater, I., Dunn, D.E., Stam, D.M., Showalter, M.R., Hammel, H.B., Min, M., Hartung, M., Gibbard, S.G., van Dam, M.A., Matthews, K., 2013. Keck and VLT AO observations and models of the uranian rings during the 2007 ring plane crossings. Icarus 226 (2), 1399-1424. http://dx.doi.org/10.1016/j.icarus.2013.08.001.

Dodson-Robinson, S.E., Bodenheimer, P., 2010. The formation of Uranus and Neptune in solid-rich feeding zones: connecting chemistry and dynamics. Icarus 207, 491-498. http://dx.doi.org/10.1016/j.icarus.2009.11.021. 
Duncan, M.J., Lissauer, J.J., 1997. Orbital stability of the uranian satellite system. Icarus 125 (1), 1-12. http://dx.doi.org/10.1006/icar.1996.5568.

Elliot, J.L., Nicholson, P.D., 1984. The rings of Uranus. In: Brahic, A., Greenberg, R. (Eds.), Planetary Rings. University of Arizona Press, Tucson.

Feuchtgruber, H., Lellouch, E., de Graauw, T., Bézard, B., Encrenaz, T., Griffin, M., 1997. External supply of oxygen to the atmospheres of the giant planets. Nature 389, $159-162$.

Feuchtgruber, H., Lellouch, E., Orton, G., de Graauw, T., Vandenbussche, B., Swinyard, B., Moreno, R., Jarchow, C., Billebaud, F., Cavalié, T., Sidher, S., Hartogh, P., 2013. The D/H ratio in the atmospheres of Uranus and Neptune from Herschel-PACS observations. Astron. Astrophys. 551, A126. http://dx.doi. org/10.1051/0004-6361/201220857.

Fienga, A., Laskar, J., Kuchynka, P., Le Poncin-Lafitte, C., Manchel, H., Gastineau, M. 2010. Gravity tests with INPOP- planetary ephemerides. In: Klioner, S.A., Seidelmann, P.K., Soffel, M.H. (Eds.), Relativity in Fundamental Astronomy. Proceedings of the IAU Symposium 261, pp. 159-169. http://dx.doi.org/10.1017/ S1743921309990330.

Fortney, J., Nettelmann, N., 2010. The interior structure, composition, and evolution of giant planets. Space Sci. Rev. 152 (1-4), 423-447. http://dx.doi.org/10.1007/ s11214-009-9582-x

French, R.S., Nicholson, P.D., Porco, C.C., Marouf, E.A., 1991. Dynamics and structure of the uranian rings. In: Bergstrahl, J.T., Miner, E.D., Matthews, M.S. (Eds.), Uranus. University of Arizona Press, Tucson, pp. 327-409.

French, R.S., Showalter, M.S., 2012. Cupid is doomed: an analysis of the stability of the inner uranian satellites. Icarus 220 (2), 911-921. http://dx.doi.org/10.1016/j. icarus.2012.06.031.

Fressin, F., Torres, G., Charbonneau, D., Bryson, S.T., Christiansen, J., Dressing, C.D. Jenkins, J.M., Walkowicz, L.M., Batalha, N.M., 2013. The false positive rate of Kepler and the occurrence of planets. Astron. Phys. J. 766, 81. http://dx.doi.org/ $10.1088 / 0004-637 X / 766 / 2 / 81$.

Fry, P.M., Sromovsky, L.A., de Pater, I., Hammel, H.B., Rages, K.A., 2012. Detection and tracking of subtle cloud features on Uranus. Astron. J. 143, 150. http://dx. doi.org/10.1088/0004-6256/143/6/150.

Grav, T., Holman, M.J., Fraser, W.C., 2004. Photometry of irregular satellites of Uranus and Neptune. Astron. J. 613, L77-L80.

Grundy, W.M., Young, L.A., Spencer, J.R., Johnson, R.E., Young, E.F., Buie, M.W., 2006. Distributions of $\mathrm{H} 2 \mathrm{O}$ and $\mathrm{CO} 2$ ices on Ariel, Umbriel, TItania, and Oberon from IRTF/SpeX observations. Icarus 184 (2), 543-555. http://dx.doi.org/10.1016/j. icarus.2006.04.016.

Guillot, T., 2005. The interiors of giant planets: models and outstanding questions. Annu. Rev. Earth Planet. Sci. 33, 493-530. http://dx.doi.org/10.1146/annurev. earth.32.101802.120325.

Guillot, T., Gautier, D., 2010. Giant planets. In: Schubert, G., Spohn, T. (Eds.), Treatise on Geophysics, vol. 10 - Planets and Moons. pp. 439-464. http://dx.doi.org/10. 1016/B978-044452748-6.00165-6.

Hammel, H.B., Lynch, D.K., Russell, R.W., Sitko, M.L., Bernstein, L.S., Hewagama, T., 2006. Mid-infrared ethane emission on Neptune and Uranus. Astron. Phys. J. 644 (2), 1326-1333. http://dx.doi.org/10.1086/503599.

Hedman, M.M., Burt, J.A., Burns, J.A., Tiscareno, M.S., 2010. The shape and dynamics of a heliotropic dusty ringlet in the Cassini Division. Icarus 210 (1), 284-297.

Hedman, M.M., Nicholson, P.D., 2013. Kronoseismology: using density waves in Saturn's C ring to probe the planet's interior. Astron. J. 146, 12. http://dx.doi. org/10.1088/0004-6256/146/1/12.

Helled, R., Anderson, J.D., Schubert, G., 2010. Uranus and Neptune: shape and rotation. Icarus 210 (1), 446-454. http://dx.doi.org/10.1016/j.icarus.2010.06.037.

Herbert, F., Sandel, B.R., Yelle, R.V., Holberg, J.B., Broadfoot, A.L., Shemansky, D.E., Atreya, S.K., Romani, P.N., 1987. The upper atmosphere of Uranus - EUV occultations observed by Voyager 2. J. Geophys. Res. 92 (A13), 15093-15109. http://dx.doi.org/10.1029/JA092iA13p15093.

Herbert, F., 2009. Aurora and magnetic field of Uranus. J. Geophys. Res. 114, A11206. http://dx.doi.org/10.1029/2009JA014394.

Hofstadter, M.H., Butler, B.J., Gurwell, M.A., 2006. Imaging of Uranus at submillimeter to centimeter wavelengths. Bull. Am. Astron. Soc. 38, 488

Holme, R., Bloxham, J., 1996. The magnetic fields of Uranus and Neptune: methods and models. J. Geophys. Res. 101 (E1), 2177-2200. http://dx.doi.org/10.1029/95JE03437.

Horányi, M., 1996. Charged dust dynamics in the solar system. Annu. Rev. Astron. Astrophys. 34, 383-418.

Horányi, M., Burns, J.A., Hamilton, D.P., 1992. The dynamics of Saturn's E ring particles. Icarus 97 (2), 248-259.

Hsu, H.-W., Postberg, F., Kempf, S., Trieloff, M., Burton, M., Roy, M., MoragasKlostermeyer, G., Srama, R., 2011. Stream particles as the probe of the dustplasma-magnetosphere interaction at Saturn. J. Geophys. Res. 116, A09215.

Hubbard, et al., 1995. The interior of Neptune, Hubbard, Podolak and Stevenson, Neptune and Triton. University of Arizona Press, Tucson, Arizona, USA, pp. 109-138.

Hubbard, W.B., 2010. Ice giants decadal study. 〈http://sites.nationalacademies.org/ SSB/SSB_059331> (Retrieved 7.10.10).

Hussmann, H., Sohl, F., Spohn, T., 2006. Subsurface oceans and deep interiors of medium-sized outer planet satellites and large trans-neptunian objects. Icarus 185 (1), 258-273. http://dx.doi.org/10.1016/j.icarus.2006.06.005.

Iafolla, V., Fiorenza, E., Lefevre, C., Morbidini, A., Nozzoli, S., Peron, R., Persichini, M., Reale, A., Santoli, F. 2010. Italian Spring Accelerometer (ISA): a fundamental support to BepiColombo Radio Science Experiments. Planet. Space Sci. 58 (1-2), 300-308. http://dx.doi.org/10.1016/j.pss.2009.04.005.

Jacobson, R.A., Campbell, J.K., Taylor, A.H., Synnott, S.P., 1992. The masses of Uranus and its major satellites from Voyager tracking data and Earth-based uranian satellite data. Astron. J. 103 (6), 2068-2078.
Jacobson, R.A., 1998. The orbits of the inner uranian satellites from Hubble Space Telescope and Voyager 2 observations. Astron. J. 115 (3), 1195-1199. http://dx. doi.org/10.1086/300263.

Jacobson, R.A., 2007. The gravity field of the uranian system and the orbits of the uranian satellites and rings. Presented at the American Astronomical Society DPS meeting \#39. Bull. Am. Astron. Soc. 39, 453.

Janes, D.M., Melosh, H.J., 1988. Sinker tectonics - an approach to the surface of Miranda. J. Geophys. Res. 93, 3127-3143. http://dx.doi.org/10.1029/ JB093iB04p03127.

anhunen, P., 2004. Electric sail for spacecraft propulsion. J. Propuls. Power 20, 763-764.

Janhunen, P., Lebreton, J.-P., Merikallio, S., Paton, M., Mengali, G., Quarta, A.A., 2014 Fast E-sail Uranus entry probe mission. Planet. Space Sci, (in this issue).

Karkoschka, E., Tomasko, M., 2009. The haze and methane distributions on Uranus from HST-STIS spectroscopy. Icarus 202 (1), 287-309. http://dx.doi.org/10.1016/ j.icarus.2009.02.010.

Kempf, S, Srama, R., Horányi, M., Burton, M., Helfert, S., Moragas-Klostermeyer, G. Roy, M., Grün, E., 2005. High-velocity streams of dust originating from Saturn. Nature 433 (7023), 289-291.

Khurana, K.K., Kivelson, M.G., Stevenson, D.J., Schubert, G., Russell, C.T., Walker, R.J. Polanskey, C., 1998. Induced magnetic fields as evidence for subsurface ocean in Europa and Callisto. Nature 395 (6704), 777-780. http://dx.doi.org/10.1038/ 27394.

Kurth, W. G, Gurnett, D.A. Coroniti, FV Scarf, F. 1991. Wave-particle interactions in the magnetosphere of Uranus. In: Bergstrahl, J.T., Miner, E.D., Matthews, M.S. (Eds.), Uranus. Umiversity of Arizona Press, Tucson.

Lainey, V., 2008. A new dynamical model for the uranian satellites. Planet. Space Sci. 56 (14), 1766-1772. http://dx.doi.org/10.1016/j.pss.2008.02.015.

Lamy, L., Prangé, R., Hansen, K.C., Clarke, J.T., Zarka, P., Cecconi, B., Aboudarham, J. André, N., Branduardi-Raymont, G., Gladstone, R., Barthélémy, M., Achilleos, N. Guio, P., Dougherty, M.K., Melin, H., Cowley, S.W.H., Stallard, T.S., Nichols, J.D. Ballester, G., 2012. Earth-based detection of Uranus' aurorae. Geophys. Res. Lett. 39, L07105. http://dx.doi.org/10.1029/2012GL051312.

Leinhardt, Z.M., Ogilvie, G.I., Latter, H.N., Kokubo, E., 2012. Tidal disruption of satellites and formation of narrow rings. MNRAS 424 (2), 1419-1431. http://dx. doi.org/10.1111/j.1365-2966.2012.21328.x.

Lissauer, J.J., 2005. Formation of the outer planets. Space Sci. Rev. 116, 11-24. http: /dx.doi.org/10.1007/s11214-005-1945-3.

Lopes, R.M.C., Kirk, R.L., Mitchell, K.L., Legall, A., Barnes, J.W., Hayes, A., Kargel, J., Wye, L., Radebaugh, J., Stofan, E.R., Janssen, M.A., Neish, C.D., Wall, S.D., Wood, C.A., Lunine, J.I., Malaska, M.J., 2013. Cryovolcanism on Titan: new results from Cassini RADAR and VIMS. J. Geophys. Res.: Planets 118, 416-435. http://dx.doi. org/10.1002/jgre.20062.

Luu, J.X., Jewitt, D.C., Trujillo, C., 2000. Water ice in 2060 Chiron and its implications for Centaurs and Kuiper belt objects. Astron. Phys. J. 531, L151-154. http://dx. doi.org/10.1086/312536.

Majeed, T., Waite, J.H., Bougher, S.W., Yelle, R.V., Gladstone, G.R., McConnell, J.C., Bhardwaj, A., 2004. The ionospheres-thermospheres of the giant planets. Adv. Space Res. 33 (2), 197-211. http://dx.doi.org/10.1016/j.asr.2003.05.009.

Maris, M., Carraro, G., Cremonese, G., Fulle, M., 2001. Multicolor photometry of the Uranus irregular satellites Sycorax and Caliban. Astron. J. 121, 2800-2803.

Maris, M., Carraro, G., Parisi, M.G., 2007. Light curves and colours of the faint Uranian irregular satellites Sycorax, Prospero, Stephano, Setebos, and Trinculo. A\&A 472, 311-319. http://dx.doi.org/10.1051/0004-6361:20066927.

Masters, A., 2014. Magnetic reconnection at Uranus' magnetopause. J. Geophys. Res. 119, http://dx.doi.org/10.1002/2014JA020077.

Matthews, M.S., Bergstralh, J.T., Miner, E.D., 1991. Uranus. University of Arizona Press, Tucson, ISBN: 978-0-8165-1208-9.

McNutt Jr, R.L., Selesnick, R.S., Richardson, J.D., 1987. Low-energy plasma observations in the magnetosphere of uranus. J. Geophys. Res. 92 (A5), 4399-4410.

Melin, H., Stallard, T., Miller, S., Trafton, L.M., Encrenaz, Th., Geballe, T.R., 2011 Seasonal variability in the ionosphere of Uranus. Astrophys. J. 729, 134. http: //dx.doi.org/10.1088/0004-637X/729/2/134.

Melin, H., Stallard, T., Miller, S., Geballe, T.R., Trafton, L.M., O’Donoghue, J., 2013. Post-equinoctial observations of the ionosphere of Uranus. Icarus 223 (2), 741-748. http://dx.doi.org/10.1016/j.icarus.2013.01.012.

Mauk, B.H., Krimigis, S.M., Keath, E.P., Cheng, A.F., Armstrong, T.P., Lanzerotti, L.J. Gloeckler, G., Hamilton, D.C., 1987. The hot plasma and radiation environment of the uranian magnetosphere. J. Geophys. Res. 92 (A13), 15283-15308.

Morbidelli, A., Tsiganis, K., Batygin, K., Crida, A., Gomes, R., 2012. Explaining why the uranian satellites have equatorial prograde orbits despite the large planetary obliquity. Icarus 219 (2), 737-740. http://dx.doi.org/10.1016/j. icarus.2012.03.025.

Moses, J.I., Fouchet, T., Bézard, B., Gladstone, G.R., Lellouch, E., Feuchtgruber, H., 2005. Photochemistry and diffusion in Jupiter's stratosphere: constraints from ISO observations and comparisons with other giant planets. J. Geophys. Res. 110, E08001. http://dx.doi.org/10.1029/2005JE002411.

Murray, C.D., Thompson, R.P., 1990. Orbits of shepherd satellites deduced from the structure of the rings of Uranus. Nature 348, 499-502. http://dx.doi.org/ $10.1038 / 348499 a 0$

Ness, N.F., Connerney, J.E.P., Lepping, R.P., Schulz, M., Voigt, G.-H., 1991. The magnetic field and magnetospheric configuration of Uranus. In: Bergstrahl, J.T., Miner. E.D. Matthews, M.S. (Eds.), Uranus. University of Arizona Press, Tucson, pp. 739-779.

Nettelmann, N., Helled, R., Fortney, J.J., Redmer, R., 2013. New indication for a dichotomy in the interior structure of Uranus and Neptune from the application of modified shape and rotation data. Planet. Space Sci. 77, 143-151. http://dx. doi.org/10.1016/j.pss.2012.06.019. 
Nojiri, S., Odintsov, S., 2007. Introduction to modified gravity and gravitationa alternative for dark energy. Int. J. Geom. Methods Mod. Phys. 4 (1), 115-145. http://dx.doi.org/10.1142/S0219887807001928.

O'Brien, R.C., Ambrosi, R.M., Bannister, N.P., Howe, S.D., Atkinson, H.V., 2008. Safe radioisotope thermoelectric generators and heat sources for space applications. J. Nucl. Mater. 377 (3), 506-521.

O’Donoghue, J.T., Stallard, S., Melin, H., Jones, G.H., Cowley, S.W.H., Miller, S., Baines, K.H., Blake, J.S.D., 2013. The domination of Saturn's low-latitude ionosphere by ring 'rain'. Nature 496 (7444), 193-195, http://dx.doi.org/10.1038/nature12049.

Owen, T., Encrenaz, T., 2006. Compositional constraints on giant planet formation. Planet. Space Sci. 54 (12), 1188-1196. http://dx.doi.org/10.1016/j.pss.2006. 05.030.

Pappalardo, R.T., Reynolds, S.J., Greeley, R., 1997. Extensional tilt blocks on Miranda: evidence for an upwelling origin of Arden Corona. J. Geophys. Res.: Planets 102 (E6), 13369-13380. http://dx.doi.org/10.1029/97JE00802.

Parisi, M.G., Carraro, G., Maris, M., Brunini, A., 2008. Constraints to Uranus' grea collision IV: the origin of Prospero. A\&A 482, 657-664. http://dx.doi.org/ 10.1051/0004-6361:20078265.

Pearl, J.C., Conrath, B.J., Hanel, R.A., Pirraglia, J.A., 1990. The albedo, effective temperature, and energy balance of Uranus, as determined from Voyager IRIS data. Icarus 84, 12-28. http://dx.doi.org/10.1016/0019-1035(90)90155-3.

Plescia, J.B., 1987. Cratering history of the Uranian satellites-Umbriel, Titania, and Oberon. J. Geophys. Res. 92, 14918-14932. http://dx.doi.org/10.1029/JA092iA13 p14918.

Postberg, F., Schmidt, J., Hillier, J.K., Kempf, S., Srama, R., 2011. A salt-water reservoir as the source of a compositionally stratified plume on Enceladus. Nature 474 (7), 620-622.

Rages, K., Pollack, J.B., Tomasko, M.G., Doose, L.R., 1991. Properties of scatterers in the troposphere and lower stratosphere of Uranus based on Voyager imaging data. Icarus 89, 359-376.

Redmer, R., Mattsson, T.R., Nettelmann, N., French, M., 2011. The phase diagram of water and the magnetic fields of Uranus and Neptune. Icarus 211, 798-803. http://dx.doi.org/10.1016/j.icarus.2010.08.008.

Reynaud, S., Jaekel, M.T., 2005. Testing the Newton law at long distances. Int. J. Mod Phys. A 20, 2294. http://dx.doi.org/10.1142/S0217751 × 05024523.

Richardson, J.D., Belcher, J.W., Selesnick, R.S., Zhang, M., Siscoe, G.L., Eviatar, A. 1988. Evidence for periodic reconnection at Uranus? Geophys. Res. Lett. 15 (8), $733-736$.

Romon, J., de Bergh, C., Barucci, M.A., Doressoundiram, A., Cuby, J.-G., Le Bras, A. Douté, S., Schmitt, D., 2001. Photometric and spectroscopic observations of Sycorax, satellite of Uranus. A\&A 376, 310-315.

Safronov, 1972. Evolution of the protoplanetary cloud and formation of the earth and planets. Translated from Russian. Jerusalem (Israel): Israel Program for Scientific Translations, Keter Publishing House, p. 212. Saumon \& Guillot (2004). Astron. Phys. J. 609(2), 1170-1180.

Sarsfield, M.J., Bell, K., Maher, C.J., Carrott, M.J., Gregson, C., Brown, J., Woodhead,D. A., Baker, S.R. , Cordingley, L., Taylor, R.J., Tinsley, T.P., Rice, T.G., Rhodes, C.J., Clough, M., 2013. Progress on ${ }^{241}$ Am production for use in radioisotope power systems. In: Proceedings of Nuclear and Emerging Technologies for Space, Albuquerque, NM, USA, February 25-28, 2013.

Saumon, D., Guillot, T., 2004. Shock compression of deuterium and the interiors of Jupiter and Saturn. Astron. Phys. J. 609, 1170-1180.

Schenk, P.M., 1991. Fluid volcanism on Miranda and Ariel - flow morphology and composition. J. Geophys. Res.: Solid Earth 96 (B2), 1887-1906. http://dx.doi.org/ 10.1029/90JB01604.

Schenk, P.M., Moore, J.M., 1995. Volcanic constructs on Ganymede and Enceladus: topographic evidence from stereo images and photoclinometry. J. Geophys. Res.: Planets 100 (E9), 19009-19022. http://dx.doi.org/10.1029/95JE01854.

Schubert, G., Hussmann, H., Lainey, V., Matson, D.L., McKinnon, W.B., Sohl, F., Sotin, C., Tobie, G., Turrini, D., van Hoolst, T., 2010. Evolution of icy satellites. Space Sci. Rev. 153 (1-4), 447-484. http://dx.doi.org/10.1007/s11214-010-9635-1.

Selesnick, R.S., Richardson, J.D., 1986. Plasmasphere formation in arbitrarily oriented magnetospheres. Geophys. Res. Lett. 13 (7), 624-627.

Selesnick, R.S., McNutt, R.L., 1987. Voyager 2 Plasma ion observations in the magnetosphere of Uranus. J. Geophys. Res. 92 (A13), 15249-15262.

Sheppard, S.S., Jewitt, D., Kelyna, J., 2005. An ultradeep survey for irregular satellites of Uranus: limits to completeness. Astron. J. 129, 518-525.

Showalter, M.R., Lissauer, J.J., French, R.G., Hamilton, D.P., Nicholson, P.D, de Pater, I., Dawson, R., 2008. HST observations of the Uranian outer ring-moon system. American Astronomical Society DPS meeting \#40 \#24.07. Bull. Am. Astron. Soc $40,431$.

Showalter, M., Lissauer, J., 2006. The second ring-moon system of Uranus: discovery and dynamics. Science 311 (5763), 973-977. http://dx.doi.org 10.1126/science.1122882.
Sittler, E.C., Ogilvie, K.W., Selesnick, R.S., 1987. Survey of electrons in the uranian magnetosphere: Voyager 2 observations. J. Geophys. Res. 92 (A13), $15263-15281$.

Soderlund, K.M., Heimpel, M.H., King, E.M., Aurnou, J.M., 2013. Turbulent models of ice giant internal dynamics: dynamos, heat transfer, and zonal flows. Icarus 224 (1), 97-113. http://dx.doi.org/10.1016/j.icarus.2013.02.014.

Spahn, F, Schmidt, J., Albers, N., Hörning, M., Makuch, M., Seiß, M., Kempf, S., Srama, R., Dikarev, V., Helfert, S., Moragas-Klostermeyer, G., Krivov, A.V., Sremčević, M. Tuzzolino, A.J., Economou, T., Grün, E., 2006. Cassini dust measurements at Enceladus and implications for the origin of the E ring. Science 311 (5), 1416-1418.

Squyres, S., et al., 2011. Vision and Voyages for Planetary Science in the Decade 2013-2022. Committee on the Planetary Science Decadal Survey; National Research Council. National Academies Press, Washington, DC, ISBN: 0-30920955-2.

Sromovsky, L.A., Fry, P.M., Hammel, H.B., de Pater, I., Rages, K.A., Showalter, M.R. 2007. Dynamics, evolution, and structure of Uranus' brightest cloud feature. Icarus 192 (2), 558-575.

Sromovsky, L.A., Fry, P.M., Hammel, H.B., Ahue, W.M., de Pater, I., Rages, K.A., Showalter, M.R., van Dam, M.A., 2009. Uranus at equinox: cloud morphology and dynamics. Icarus 203 (1), 265-286. http://dx.doi.org/10.1016/j.icarus. 2009.04.015.

Sromovsky, L.A., Karkoschka, E., Fry, P.M., Hammel, H.B., de Pater, I., Rages, K., 2014 Methane depletion in both polar regions of Uranus inferred from HST/STIS and Keck/NIRC2 observations. Icarus 238, 137-155. http://dx.doi.org/10.1016/j. icarus.2014.05.016.

Stanley, S., Bloxham, J., 2004. Convective-region geometry as the cause of Uranus' and Neptune's unusual magnetic fields. Nature 428 (6979), 151-153. http://dx. doi.org/10.1038/nature02376.

Stanley, S., Bloxham, J., 2006. Numerical dynamo models of Uranus' and Neptune's magnetic fields. Icarus 184 (2), 556-572. http://dx.doi.org/10.1016/j. icarus.2006.05.005.

Stone, E.C., Miner, E.D., 1986. The Voyager 2 Encounter with the Uranian System. Science 233 (4759), 39-43. http://dx.doi.org/10.1126/science.233.4759.39.

Tiscareno, M.S., 2013. Planetary rings. In: Oswalt, French and Kalas (Eds.), Planets, Stars and Stellar Systems. Springer. 〈arXiv:1112.3305〉.

Tittemore, W.C., Wisdom, J., 1990. Tidal evolution of the Uranian satellites. IIIevolution through the Miranda-Umbriel 3:1. Miranda-Ariel 5:3, and ArielUmbriel 2:1 mean-motion commensurabilities. Icarus 85, 394-443. http://dx. doi.org/10.1016/0019-1035(90)90125-S.

Tittemore, W.C., 1990. Tidal heating of Ariel. Icarus 87, 110-139. http://dx.doi.org/ 10.1016/0019-1035(90)90024-4.

Tosi, F., Turrini, D., Coradini, A., Filacchione, G., 2010. Probing the origin of the dark material on Iapetus. MNRAS 403, 1113-1130. http://dx.doi.org/10.1111/j.13652966.2010.16044.x.

Tóth, G., Kovács, D., Hansen, K.C., Gombosi, T.I., 2004. Three-dimensional MHD simulations of the magnetosphere of Uranus. J. Geophys. Res. 109, A11210. http: //dx.doi.org/10.1029/2004JA010406.

Trafton, L.M., Miller, S., Geballe, T.R., Tennyson, J., Ballester, G.E., 1999. H2 Quadrupole and $\mathrm{H} 3+$ emission from Uranus: the Uranian thermosphere, ionosphere, and aurora. Astrophys. J. 524 (2), 1059-1083. http://dx.doi.org/ $10.1086 / 307838$

Turrini, D., Magni, G., Coradini, A., 2011. Probing the history of solar system through the cratering records on Vesta and Ceres. MNRAS 413 (4), 2439-2466. http://dx. doi.org/10.1111/j.1365-2966.2011.18316.x.

Turyshev, S.G., 2008. Experimental tests of General Relativity. Annu. Rev. Nucl. Part. Sci. 58 (1), 207-248. http://dx.doi.org/10.1146/annurev.nucl.58.020807.111839.

Vasyliuñas, V.M., 1986. The convection-dominated magnetosphere of Uranus. Geophys. Res. Lett. 13, 621-623. http://dx.doi.org/10.1029/GL013i007p00621.

Vilas, F., Lederer, S.M., Gill, S.L., Jarvis, K.S., Thomas-Osip, J.E., 2006. Aqueous alteration affecting the irregular outer planets satellites: evidence from spectral reflectance. Icarus 180, 453-463.

Walsh, K.J., Morbidelli, A., Raymond, S.N., O’Brien, D.P., Mandell, A.M., 2011. A low mass for Mars from Jupiter's early gas-driven migration. Nature 475 (7355), 206-209. http://dx.doi.org/10.1038/nature10201.

Zarka, P., Lecacheux, A., 1987. Beaming of uranian nightside kilometric radio emission and inferred source location. J. Geophys. Res. 92, 15177-15187. http: //dx.doi.org/10.1029/JA092iA13p15177.

Zarka, P., 2007. Plasma interactions of exoplanets with their parent star and associated radio emissions. Planet. Space Sci. 55 (5), 598-617. http://dx.doi. org/10.1016/j.pss.2006.05.045. 